\title{
Dark matter in the Milky Way
}

\section{The Hı gas distribution as a tracer of the gravitational potential}

\author{
P. M. W. Kalberla ${ }^{1}$, L. Dedes ${ }^{1}$, J. Kerp ${ }^{1}$, and U. Haud ${ }^{2}$ \\ 1 Argelander-Institut für Astronomie, Universität Bonn`, Auf dem Hügel 71, 53121 Bonn, Germany \\ e-mail: [pkalberla; ldedes; jkerp]@astro.uni-bonn.de \\ 2 Tartu Observatory, 61602 Toravere, Estonia \\ e-mail: urmas@aai.ee
}

Received 8 September 2006 / Accepted 20 March 2007

\section{ABSTRACT}

\begin{abstract}
Context. Gas within a galaxy is forced to establish pressure balance against gravitational forces. The shape of an unperturbed gaseous disk can be used to constrain dark matter models.

Aims. We derive the 3D H I volume density distribution for the Milky Way out to a galactocentric radius of $40 \mathrm{kpc}$ and a height of $20 \mathrm{kpc}$ to constrain the Galactic mass distribution.

Methods. We used the Leiden/Argentine/Bonn all sky 21-cm line survey. The transformation from brightness temperatures to densities depends on the rotation curve. We explored several models, reflecting different dark matter distributions. Each of these models was set up to solve the combined Poisson-Boltzmann equation in a self-consistent way and optimized to reproduce the observed flaring. Results. Besides a massive extended halo of $M \sim 1.8 \times 10^{12} M_{\odot}$, we find a self-gravitating dark matter disk with $M=2$ to $3 \times 10^{11} M_{\odot}$, including a dark matter ring at $13<R<18.5 \mathrm{kpc}$ with $M=2.2$ to $2.8 \times 10^{10} M_{\odot}$. The existence of the ring was previously postulated from EGRET data and coincides with a giant stellar structure that surrounds the Galaxy. The resulting Milky Way rotation curve is flat up to $R \sim 27 \mathrm{kpc}$ and slowly decreases outwards. The H I gas layer is strongly flaring. The $H W H M$ scale height is $60 \mathrm{pc}$ at $R=4 \mathrm{kpc}$ and increases to $\sim 2700 \mathrm{pc}$ at $R=40 \mathrm{kpc}$. Spiral arms cause a noticeable imprint on the gravitational field, at least out to $R=30 \mathrm{kpc}$.

Conclusions. Our mass model supports previous proposals that the giant stellar ring structure is due to a merging dwarf galaxy. The fact that the majority of the dark matter in the Milky Way for $R \lesssim 40 \mathrm{kpc}$ can be successfully modeled by a self-gravitating isothermal disk raises the question of whether this massive disk may have been caused by similar merger events in the past. The substructure in the Galactic dark matter disk suggests a dissipative nature for the dark matter disk.
\end{abstract}

Key words. Galaxy: disk - Galaxy: structure - Galaxy: kinematics and dynamics - galaxies: interactions - ISM: structure Galaxy: halo

\section{Introduction}

The shape of the Galactic H I disk is interesting for its own sake. Size matters and we would like to know how the Milky Way compares to other galaxies. When trying to convert the observed brightness temperature distribution to a volume density distribution one realizes, however, that this transformation depends on the shape of the rotation curve. Determining the rotation curve needs accurate independent measurements of velocities and distances for a broad distance range, a daunting task. The first large-scale map of the H I volume density distribution in the Milky Way (Westerhout 1957) was therefore generated from a mass model (Schmidt 1956). For this model, the rotation curve at distances $R \gtrsim 10 \mathrm{kpc}$ is falling.

Observations, however, give opposite results. Georgelin \& Georgelin (1976) were the first to notice a rising rotation curve for a relatively large sample. They used $\mathrm{H} \alpha$ radial velocities and distances of stars that excite Galactic $\mathrm{H}$ II regions and found an increase of $v_{\text {rot }}$ for $R \gtrsim 10 \mathrm{kpc}$. Additional systematical variations in $v_{\text {rot }}$ for the northern and southern parts of the Galaxy led

* Founded by merging of the Sternwarte, Radioastronomisches Institut and Institut für Astrophysik und Extraterrestrische Forschung der Universität Bonn. to the question of whether a rotation curve from an axisymmetric model suffers from systematic errors.

The discrepancy between mass models and observations has persisted until recently. Theoretical mass models predict a falling rotation curve (e.g. Bahcall \& Soneira 1980; Caldwell \& Ostriker 1981; Rohlfs \& Kreitschmann 1981; Haud \& Einasto 1989; Dehnen \& Binney 1998) while observations have consistently indicated the opposite (e.g. Blitz 1979; Schneider \& Terzian 1983; Fich et al. 1989; Merrifield 1992; Brand \& Blitz 1993; Honma \& Sofue 1997).

The situation is further complicated by the fact that the rotation curve depends on the choice of Galactic constants. A high rotational velocity at the position of the Sun leads to a rising rotation curve, while the opposite holds for a low velocity. The interrelation between Galactic constants, dark matter models, and the shape of the $\mathrm{H}$ I distribution has been studied in a series of papers by Olling \& Merrifield $(1998,2000,2001)$. These authors constrain the Galactic constants to $R_{\odot} \lesssim 8 \mathrm{kpc}$ and $v_{\odot} \lesssim 200 \mathrm{~km} \mathrm{~s}^{-1}$.

Rohlfs \& Kreitschmann (1988) considered models with a rising rotation curve for $11 \lesssim R \lesssim 18 \mathrm{kpc}$ but found it difficult to explain this by a dark corona. Such a solution would result in a high rotational velocity of $400 \lesssim v_{\text {rot }} \lesssim 500 \mathrm{~km} \mathrm{~s}^{-1}$ outside the Galactic disk, which appears highly implausible. In addition, 
such a dark matter distribution would disable an exponential stellar disk, which conflicts with observations.

Binney \& Dehnen (1997) discuss the question of whether distance errors may mimic a rising rotation curve. They propose that most of the tracers that appear to be at $R \gtrsim 11 \mathrm{kpc}$ might actually be concentrated within a ring at $R \sim 14 \mathrm{kpc}$. The resulting rotation curve would be roughly constant or gently falling. Several groups - Newberg et al. (2002); Ibata et al. (2003); Yanny et al. (2003); Martin et al. (2005); Martínez-Delgado et al. (2005); Bellazzini et al. (2006) - have subsequently claimed to have detected stellar streams in the Milky Way, right at the region of interest. A ring-like mass concentration within this area plays an important role throughout our paper.

The IAU-recommended Galactic constants $R_{\odot}=8.5 \mathrm{kpc}$ and $v_{\odot}=220 \mathrm{~km} \mathrm{~s}^{-1}$ fit best with a flat rotation curve (Fich et al. 1989). Typical Sb galaxies have nearly flat outer rotation curves (Sofue \& Rubin 2001). It seems to be an acceptable compromise to assume that the Milky Way is typical in this respect. For about a decade now there has been little discussion about the Galactic rotation curve. A flat rotation curve with $v_{\text {rot }}=220 \mathrm{~km} \mathrm{~s}^{-1}$ was used for the most recent determination of the Galactic volume density distribution by Levine et al. (2006a).

The need to obtain better constraints for the radial mass distribution in the Milky Way has been stressed by many authors. Gas can play a particularly interesting role as a tracer of the gravitational potential, provided that its velocity dispersion is position-independent and that there are no external forces. The inner part of a galactic disk is dominated by stars. This results in strong gravitational forces $k_{z}$ perpendicular to the disk. The influence of the stars decreases with increasing galactocentric distance $R$. That allows the gas, which withstands the forces by means of internal turbulent motions, to reach larger scale heights. The H I disks in galaxies are typically three times more extended than their stellar counterparts. This property, as well as the fact that the outer parts of galaxies are dominated by dark matter, highlights the importance of the gaseous disk as a tracer of the mass distribution (Sofue \& Rubin 2001; Combes 2002). The Galactic outskirts are very interesting in this respect.

Gaseous flaring was first used as a diagnostic of halo properties by Olling (1995), who showed that the halo of NGC 4244 was highly flattened. Becquaert \& Combes (1997) obtained a comparable result for NGC 891. Applying a similar analysis to the Milky Way, however, has led to conflicting results (Olling $\&$ Merrifield 1998, 2000, 2001). Narayan et al. (2005) show that neither an oblate nor a prolate isothermal halo can explain the Milky Way flaring. They advocate a steeper fall-off for the halo density.

Surprisingly, little is known about the Galactic gas- and mass distribution at large radial distances. Our knowledge is limited to distances $R \lesssim 22 \mathrm{kpc}$ (Binney \& Merrifield 1998, and references therein). The Galactic outskirts are extremely sensitive to the dark matter distribution. The main driver of the present paper is therefore to explore the mass distribution at large radial distances. A second, even more severe problem has been pointed out by Dehnen \& Binney (1998). There are large observational uncertainties in the mass distribution perpendicular to the disk. Constraints at $z \gtrsim R$ are required. The gravitational acceleration $k_{z}$ in the solar vicinity is well known from stellar statistics up to distances of $z \lesssim 1.5 \mathrm{kpc}$ (Kuijken \& Gilmore 1991; Holmberg \& Flynn 2000; Korchagin et al. 2003; Holmberg \& Flynn 2004), but little is known at larger distances. Figure 11 of Holmberg \& Flynn (2004) impressively demonstrates that observational constraints are missing from star counts. Extra-planar H I gas, though faint, may be a better probe since it extends to large $z$ distances (Lockman \& Gehman 1991; Kalberla et al. 1998).

This paper is organized in the following way: Sect. 2 describes our data analysis, in particular the methods used to derive the H I flaring. In Sect. 3 we discuss how mass models affect the derived $\mathrm{H}$ I volume density distribution and flaring. Each mass model is checked for consistency. The rotation curve associated with the best-fit model is discussed in Sect. 4. We consider further consistency checks for our model, using flaring data from the literature and HIPPARCOS surface densities in Sects. 5 and 6. The warp parameters are summarized in Sect. 7. We discuss deviations by the Milky Way flaring from the axisymmetric model and spiral structures in Sect. 8. Uncertainties and possible biases due to model assumptions are considered in Sect. 9. The results are summarized in Sect. 10. We discuss the nature of the dark matter ring and disk in Sect. 11.

\section{Data analysis and methods}

Our study is based on the Leiden/Argentine/Bonn (LAB) H I line survey (Kalberla et al. 2005). This survey combines the southern sky survey of the Instituto Argentino de Radioastronomía (IAR) (Bajaja et al. 2005) with an improved version of the Leiden/Dwingeloo Survey (LDS) (Hartmann \& Burton 1997). Currently, this is the most sensitive Milky Way H I line survey with the most extensive coverage both spatially and kinematically. The line profiles have been corrected for spurious sidelobe emission. Low-level emission features can be interpreted safely as tracers of Galactic structure out to large distances, and we intend to explore the outskirts of the Milky Way.

\section{1. $T_{B}(I, b, v)$ to $n(R, z, \phi)$ conversion}

We use cylinder coordinates $R, z, \phi$ to describe the Milky Way disk; $\phi=0$ is in direction $l=0$. We use the IAU recommendations for the galactic constants; $R_{\odot}=8.5 \mathrm{kpc}$ and $v_{\odot}=$ $220 \mathrm{~km} \mathrm{~s}^{-1}$. All the individual steps necessary for converting the observed brightness temperature distribution $T_{B}(l, b, v)$ as a function of galactic longitude, latitude, and LSR velocity to densities $n(R, z, \phi)$ have been described before in great detail, e.g., by Westerhout (1957); Henderson et al. (1982); Burton \& Te Lintel Hekkert (1986); Diplas \& Savage (1991); Voskes (1999); Nakanishi \& Sofue (2003) and Levine et al. (2006a). We sketch our procedure only briefly; but where we deviate from other authors, we discuss those points in detail.

Previously, Levine et al. (2006a) have used the Hanning smoothed version of the LAB survey to derive $n(R, z, \phi)$. Our approach is similar, but we made use of the original telescope data, cleaned for instrumental problems (the version prior to the generation of FITS maps; Kalberla et al. 2005).

To derive volume densities $n(R, z, \phi)$ for each volume element centered at $R_{0}, z_{0}, \phi_{0}$ we calculated the emission within this cell. We first determined which of the observed $T_{B}(l, b, v)$ profiles may show some contribution from this volume element. For each profile we calculated column densities along the path length within the cell under consideration. The volume density was obtained after division by the path length. The procedure is similar to the one used by Diplas \& Savage (1991). We assumed that the gas is optically thin. This may, however, be incorrect for some parts of the Galactic disk at low latitudes (Burton et al. 1992).

The LAB data are expected to predominantly consist of emission from the Galactic H I disk, gas in a roughly circular motion. But there are contributions from the local gas, from galaxies, also from high- and intermediate velocity clouds (HVCs and 
IVCs, respectively), which are not directly connected to the disk. Different attempts have been made to exclude such features from the data base right at the beginning of the data processing. Some authors have interpolated and subtracted the local H I emission. Automated filtering of the $T_{B}(l, b, v)$ data may cause unknown biases. We decided not to filter the observational data base but rather the $n(R, z, \phi)$ distribution. The details for our procedure are given in Sect. 2.2.

Most important for the conversion of $T_{B}(l, b, v)$ to $n(R, z, \phi)$ is the translation of velocities to distances via a rotation curve. The exact shape of the Milky Way rotation curve is controversial, so some assumptions are needed to overcome this deficit. Any prejudice at this point may bias the results, so we decided to study how different rotation curves, corresponding to different mass models, may affect the results. We also used a generalized rotation law $v_{\text {rot }}(R, z)$, allowing the azimuthal streaming of the gas above the disk to deviate from the rotation of the disk.

A strictly circular motion of a particle at a constant $z$ distance above the disk is not possible. We considered the properties of a gas layer and describe the average rotation for a volume element with constant average volume density in a multiphase medium. We assumed that the streaming velocity $\bar{v}_{\text {rot }}$ is dominated by gravitational forces

$\bar{v}_{\text {rot }}(R, z)=\sqrt{R \partial \Phi(R, z) / \partial R}$.

Such a relation implies, for constant galactocentric radius $R$, a decreasing rotation velocity with increasing $z$ distance. It was used for the first time by Westerhout (1957) to derive the Galactic H I volume density distribution, but later publications have assumed cylindrical rotation.

In comparing cylindrical with lagging rotation we recall the conditions that must apply for the cylindrical case (Kalberla 2003, Paper I): the density distribution has to be barotropic, $p=f(\rho)$. A barotropic fluid is an idealized fluid in which the pressure is a function of only the density. The interstellar medium, however, is dominated by several co-existing phases. Here we need to consider at least two distinct $\mathrm{H}$ I phases, a cold neutral medium, and a warm neutral medium (CNM and WNM, respectively). These have very different densities but are basically in pressure equilibrium (Wolfire et al. 2003); and phase transitions are possible. Barotropic conditions do not apply to such a multi-phase medium, which probably dominates most of the volume under consideration. It is therefore highly questionable whether the common assumption of cylindrical rotation applies for the H I gas in the Milky Way disk. We therefore exercise the option of using both the cylindrical and lagging rotations for our reduction. For a more detailed discussion, see Sect. 4.1.

Except for replacing $v_{\text {rot }}(R)$ by $v_{\text {rot }}(R, z)$, we used the procedure as described in Levine et al. (2006a, Eqs. (1) and (2)) including their first order epicyclic streamline correction; which is a great improvement over previous distance derivations and important for overcoming discontinuities at large radial distances. No streamline correction was applied to $R<8.5 \mathrm{kpc}$, since the correction is not applicable there. Inside the solar circle the translation between distance and velocity is ambiguous because there are two positions along the line of sight that have the same velocity. These positions, however, may have different $z$ distances, so their densities may differ accordingly.

We modeled the $\mathrm{H}$ I distribution, taking the expected flaring according to the mass model into account. Incrementing along the line of sight, we calculated expected densities at near and far distances and weighted the derived quantities accordingly. Our method is comparable to that of Nakanishi \& Sofue (2003), except that they use a $\operatorname{sech}^{2}$ approach according to Spitzer (1942) in place of a more detailed model. We applied no correction for densities around the tangent points, as described by Nakanishi \& Sofue (2003, Sect. 3.2) since this tends to cause discontinuities. The focus of our paper is on data at $R>8.5 \mathrm{kpc}$. However, as data inside the solar circle are found to be useful for checking the consistency of a model, they are included in most of the plots.

The $n(R, z, \phi)$ distribution was calculated and interpreted for $0<R<40 \mathrm{kpc},-15<z<20 \mathrm{kpc}$ at a resolution of $\Delta R=100 \mathrm{pc}, \Delta z=100 \mathrm{pc}$, and $\Delta \phi=1^{\circ}$. In addition we allowed $l, b, v$ to be traced back for each position $R, z, \phi$. To avoid biases due to local $\mathrm{H}$ I features we flagged and discarded all data with $|b|>30^{\circ}$. Similarly we flagged data with $\left|v_{\mathrm{LSR}}\right|<10 \mathrm{~km} \mathrm{~s}^{-1}$. In the direction of the Galactic center, we discarded data originating from $|R|<3.5 \mathrm{kpc}$. This region, approximately with the extent of the bar, is affected by ambiguities due to large velocity dispersions (Weiner \& Sellwood 1999, Fig. 8).

\subsection{Determination of the first moments}

To derive genuine disk gas we initially flagged all data for $|b|>30^{\circ}$. But then we did not discard any features of the observed spectra to distinguish between gas within the disk and gas that is, most probably, not associated with the disk. The derived volume density distribution contains both contributions. An inspection of the 3D distribution shows that it is relatively easy to distinguish between both contributions visually, if one generates video sequences. The disk component, in circular motion, stays mostly stationary, while there are other components that appear to move toward the Sun as the frames approach the Sun or, opposite, as one moves away from the Sun. Galaxies, in particular, show up as fast-moving isolated spots. This effect is most prominent if the video frames loop in azimuth angle $\phi$.

To focus on the stationary disk emission, we averaged $n(R, z, \phi)$ over $15^{\circ}$ in $\phi$. This average does not have a major effect on the stationary disk component but degrades spurious emission caused by H I gas in non-circular motion considerably. We therefore used it as an initial guess of the first three moments; the surface density $\Sigma$, the first moment $z_{0}$, representing mid-plane, and the second moment $\sigma$, representing the scale height of the gas. As mentioned before, data with $|b|>30^{\circ}$, $\left|v_{\text {LSR }}\right|<10 \mathrm{~km} \mathrm{~s}^{-1}$, had been flagged in the first place. In addition, we flagged all data in the original data base outside the range $z_{0}-2.35 \sigma<z<z_{0}+2.35 \sigma$. For a Gaussian distribution, $2 \%$ of the data would be discarded by this criterion. On average we find more than double that amount. As a final step we determined the moments $\Sigma(R, \phi), z_{0}(R, \phi)$, and $\sigma(R, \phi)$ from those parts of the unsmoothed data that was not flagged, thus excluding extra-planar gas.

We verified each of the reduction steps by inspecting the data cubes visually. Most critical is the flagging process. We found the automated procedure worked as expected for a majority of cases. However, many features exist that provide no clear evidence whether or not they belong to the disk. Figures 1 and 2 show example maps for the volume density distribution at azimuth $\phi=110^{\circ}$ (right hand side) and opposite, $\phi=290^{\circ}$ (left hand side). Most of the flagging of extra-planar gas starts already in regions below $|b| \lesssim 30^{\circ}$. The latitude limit $|b|=30^{\circ}$, naively introduced to blank out local gas at high latitudes, gets justified this way and does not affect our analysis in a quantitative way.

Figure 1 displays volume densities for our best-fit model, while Fig. 2 was derived from the standard model with a constant rotation curve; for a more detailed discussion of the differences we refer the reader to Sect. 4.1. Prominent in Fig. 1 is the elongated feature at $12.5 \lesssim R \lesssim 16.5 \mathrm{kpc}$ and $4.3 \lesssim z \lesssim 6.3 \mathrm{kpc}$. 
az: $1.100000 \mathrm{e}+02$

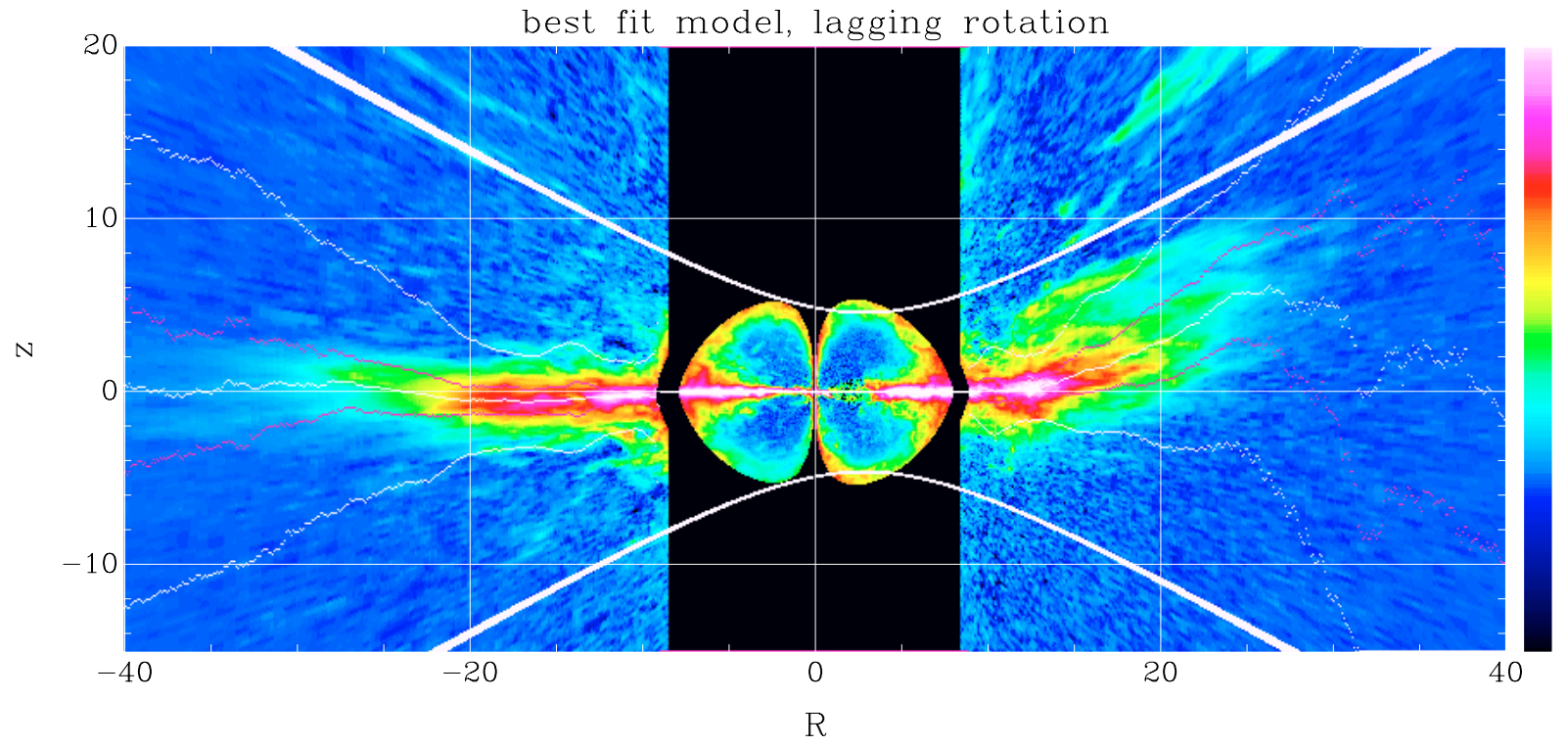

Fig. 1. Derived volume density distribution for our best-fit model at azimuth $\phi=110^{\circ}$ (right hand side) and opposite, $\phi=290^{\circ}$ (left hand side) for $0<R<40 \mathrm{kpc}$ and $-15<z<20 \mathrm{kpc}$. Densities affected by local emission with $\left|v_{\mathrm{LSR}}\right|<10 \mathrm{~km} \mathrm{~s}^{-1}$ are flagged black. The curved white, thick lines indicate latitudes $|b|=30^{\circ} \pm 0.5^{\circ}$. The derived mid plane of the $\mathrm{HI}$ distribution is indicated by the thin white line close to $z=0 \mathrm{kpc}$, and the red dots represent the dispersion of the flaring $\mathrm{HI}$ gas layer. The outer white dots indicate the initial estimate for the extension of H I gas considered to belong to the disk. A logarithmic transfer function was chosen for $n<0.5 \mathrm{~cm}^{-3}$ to emphasize low densities.

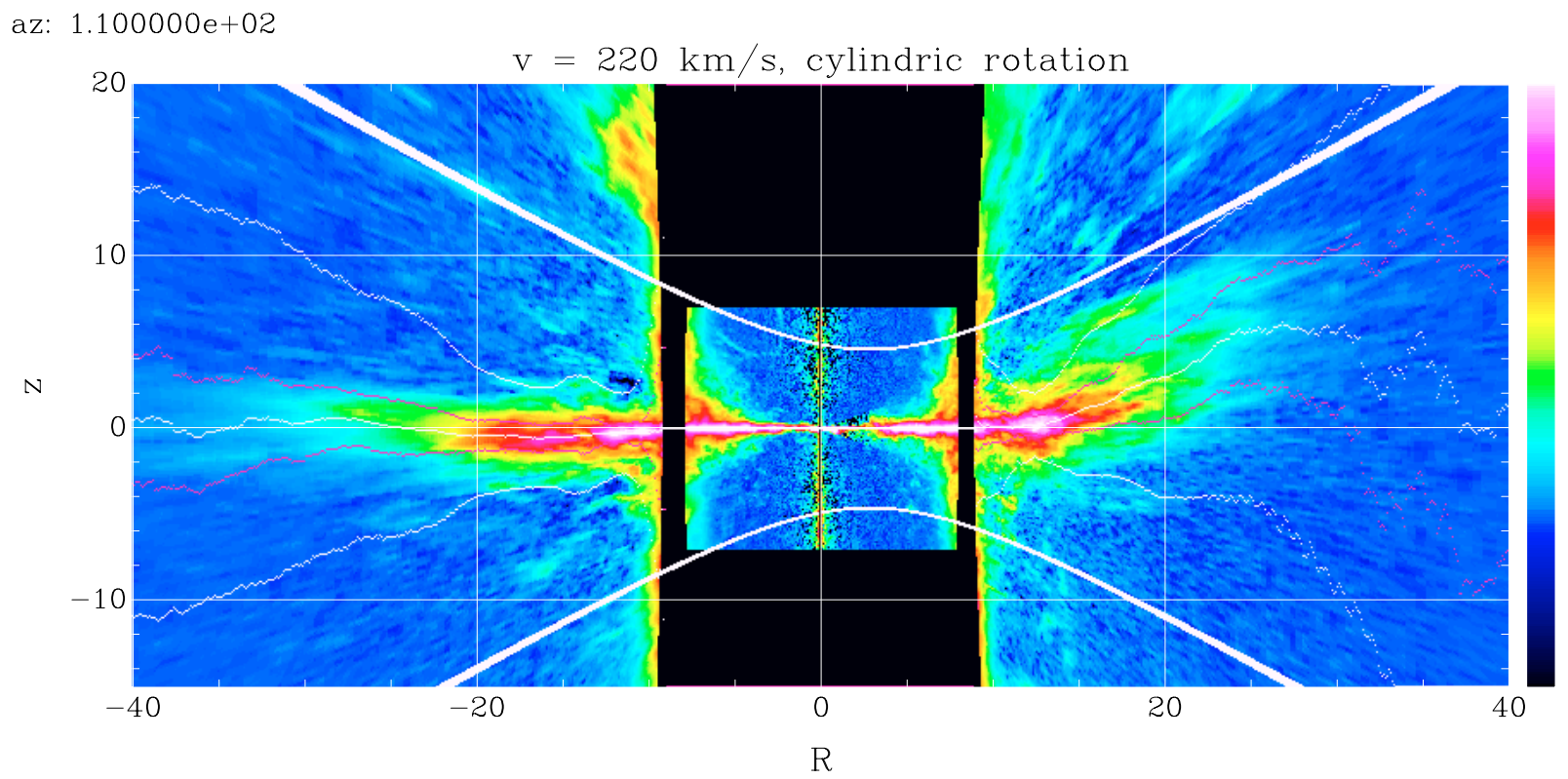

Fig. 2. Volume density distribution derived under the assumption of a constant cylindrical rotation with $v_{\text {rot }}=220$. See Fig. 1 for further explanation.

This is the HVC complex R located at $62^{\circ} \lesssim l \lesssim 73^{\circ}$ and $5.5 \lessgtr$ $b \lesssim 15^{\circ}$ with radial velocities of $-156 \lesssim v_{\mathrm{LSR}} \lesssim-100 \mathrm{~km} \mathrm{~s}^{-1}$. There are some indications that this HVC complex may be interacting with the disk (Kalberla \& Haud 2006), but this feature cannot be considered part of the stationary galactic disk. It was removed by our automatic flagging. Similarly, some components showing up in $T_{B}(l, b, v)$ as intermediate velocity clouds (IVCs) were removed by the flagging.

Figures 1 and 2 are examples only, but they do show clearly that warp and flaring of the $\mathrm{H}$ I disk are asymmetric. In particular, as discussed in Sects. 8 and 9 in more detail, the region $90^{\circ} \lesssim$ $\phi \lesssim 110^{\circ}$ appears strongly perturbed for $R \gtrsim 15 \mathrm{kpc}$. For this reason we exclude this azimuth range in the following whenever we determine average properties of the $\mathrm{HI}$ distribution.

Finally, we emphasize that our data reduction is fully automated, without any human interaction in the flagging process. The procedures were identical for all the mass models and rotation curves we considered.

\section{Mass models and volume density distributions}

Probably the most frequently used rotation law for the Milky Way assumes a constant rotation $v_{\text {rot }}(R)=220 \mathrm{~km} \mathrm{~s}^{-1}$ (Brand \& Blitz 1993). This naive model is completely free of 
specific assumptions about the dark matter distribution. We use it for reference.

We display in Fig. 2 the volume density distribution derived from the reference model at azimuth $\phi=110^{\circ}$. The most prominent features, considered to belong to the disk, are in good agreement with Fig. 1 for our best-fit model. It is the changes for HVC complex R that are remarkable. This feature has almost disappeared. The main emission from this HVC complex has shifted to an azimuth of $104^{\circ}$, while at the same time the distance in $R$ has increased by $2 \mathrm{kpc}$, in $z$ by $1 \mathrm{kpc}$.

\subsection{Definition of self-consistent mass models}

Our mass model contains the following components: an isothermal stellar disk, represented by thin and thick disk components, a bar and a bulge and isothermal gaseous disks for molecular gas, the cold neutral medium, the warm neutral medium, diffuse ionized gas (DIG), a hot ionized, and a cold neutral halo component. Surface densities and scale heights for all of these components are adjusted to match the observational constraints (we refer to Paper I for details). Such a distribution can be supplemented by any dark matter distribution that matches known observational constraints. Most importantly it must be consistent with the dark matter distribution on large scales (Zaritsky 1999). As a constraint for isothermal dark-matter halo models, we demand a total mass of $\sim 2 \times 10^{12} M_{\odot}$ within $R \lesssim 350 \mathrm{kpc}$. We demand $v_{\odot}=220 \mathrm{~km} \mathrm{~s}^{-1}$ for the local dark matter distribution.

The mass distribution for each model is checked for selfconsistency as described in Paper I (it has to solve the combined Poisson-Boltzmann equation). The visible mass distribution (stars and gas) is allowed to adjust its scale height to the total gravitational potential from disk and halo until convergence is achieved. The model disk is flat, but for comparison with observations, we applied a coordinate transformation to take warping into account (Eq. (4)).

\subsection{Self-consistent HI flaring curves}

For each mass model one has to verify whether it is consistent with the derived $\mathrm{HI}$ distribution. In each case we determined the rotation curve, converted $T_{B}(l, b, v)$ to $n(R, z, \phi)$ and calculated the moments $\Sigma(R, \phi), z_{0}(R, \phi)$, and $\sigma(R, \phi)$. We then compared the average flaring $h(R)=f\langle\sigma(R, \phi)\rangle$, derived from the data, with the expected flaring. For convenience we used for the gaseous flaring $f=\sqrt{2 \ln 2}$ to describe the half width at half maximum (HWHM) of the H I layer. This convention allows an easy comparison with the literature values.

\subsubsection{Flaring of the $\mathrm{v}_{\text {rot }}=220 \mathrm{~km} \mathrm{~s}^{-1}$ reference model}

Figure 3 shows the flaring curve derived for a flat, $v_{\text {rot }}=$ $220 \mathrm{~km} \mathrm{~s}^{-1}$ rotation curve. We excluded $90^{\circ} \lesssim \phi \lesssim 110^{\circ}$ and those regions in direction of the center or anti-center that might be affected by spurious emission not connected with the H I disk. To allow a comparison of our flaring data with previous determinations we include in Fig. 3 some data from the literature. The data for $R<R_{\odot}(\bigcirc)$ were derived from Celnik et al. (1979) by averaging over ten positions from their Table 3 . The literature data agree well with our results, except for $7 \lesssim R \lesssim 11 \mathrm{kpc}$. Our analysis is insensitive within this range.

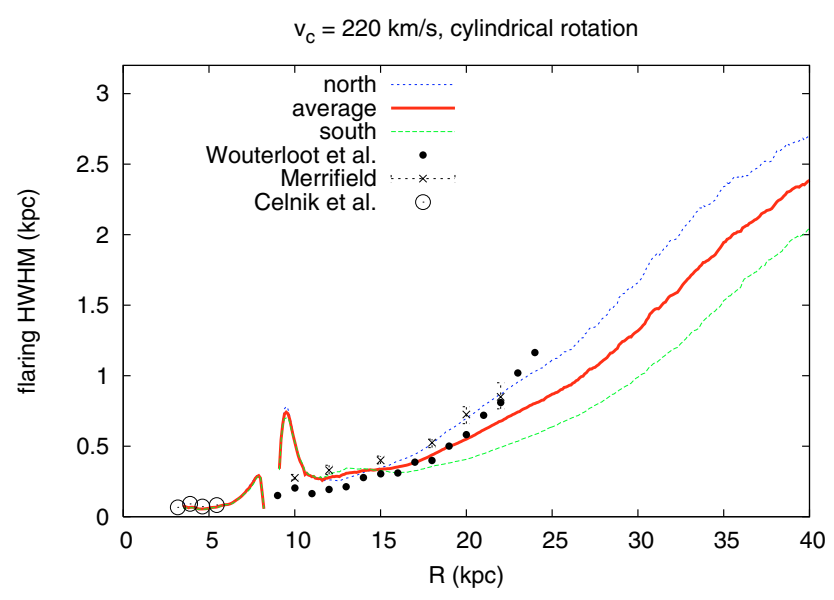

Fig. 3. Derived H I flaring for the standard model. The thick red line gives the mean extension of the flaring $\mathrm{H}$ I layer (half width at half maximum). The dotted blue and green lines give the flaring in the northern and southern hemispheres, respectively. From the literature we include: $\bigcirc$ derived from Celnik et al. (1979), $\times$ with error bars from Merrifield (1992), and • Wouterloot et al. (1990, Table 1).

\subsubsection{Flaring caused by a spheroidal halo}

We tested several conventional halo models, some of which were already discussed in Paper I. First we discuss non-singular spheroids with flattening $q$ (see e.g. Olling \& Merrifield 2001; Narayan et al. 2005),

$\rho(R, z)=\rho_{0}\left[\frac{R_{\mathrm{c}}^{2}}{R_{\mathrm{c}}^{2}+R^{2}+(z / q)^{2}}\right]^{p}$.

Oblate halos have $q<1$, prolate $q>1$. $\rho_{0}$ is the density at the center, $R_{\mathrm{c}}$ defines the core radius, and $p$ is the power index (Narayan et al. 2005). We consider the case $p=1$ first.

Figure 4 shows the $\mathrm{HI}$ flaring derived in a self-consistent way from the LAB survey for a spheroidal halo with $q=1$ in comparison to flaring curves expected for mass models with various $q$ parameters. We discuss $q=1$ first. For $R \gtrsim R_{\odot}$ the predicted flaring increases linearly with distance, in agreement with the analytical solution given by Olling \& Merrifield (2000, Eq. (8)). Comparing the model with the data, we find a systematic S-shaped deviation. The model curve explains the average trend but fails to reproduce details of the flaring derived from the data around $R \sim 17 \mathrm{kpc}$ and $R \sim 35 \mathrm{kpc}$.

To improve the model we explored how the flaring might be affected by the flattening parameter $q$. According to Olling \& Merrifield (2000), we expected changes in slope. Oblate halos, $q<1$, lead to a low flaring at $R \sim 17 \mathrm{kpc}$ but fail to reproduce the observations at $R \sim 35 \mathrm{kpc}$. Prolate halos improve considerably for $q \gtrsim 8$ but cannot reproduce the data at $R \sim 17 \mathrm{kpc}$. Each halo model also leads to a different flaring curve when fitted to the observations. Since each model leads, iteratively, to a different result for the rotation curve, there would be a need for each $q$ parameter to also have a plot for the flaring, as derived from the data. For reasons of conciseness we refrain from showing all. Changes are in any case less significant than the changes in the model's flaring curves plotted in Fig. 4.

Previous determinations of the flattening parameter $q$ from $\mathrm{HI}$ flaring led to contradictory results. Olling \& Merrifield (2000, 2001) consider the H I flaring at $R=2 R_{\odot}$ and advocate $q=0.8$. They conclude that significantly flattened halos are possible only in the case that the distance of the Sun from the Galactic center is smaller than $6.8 \mathrm{kpc}$. Narayan et al. (2005) 


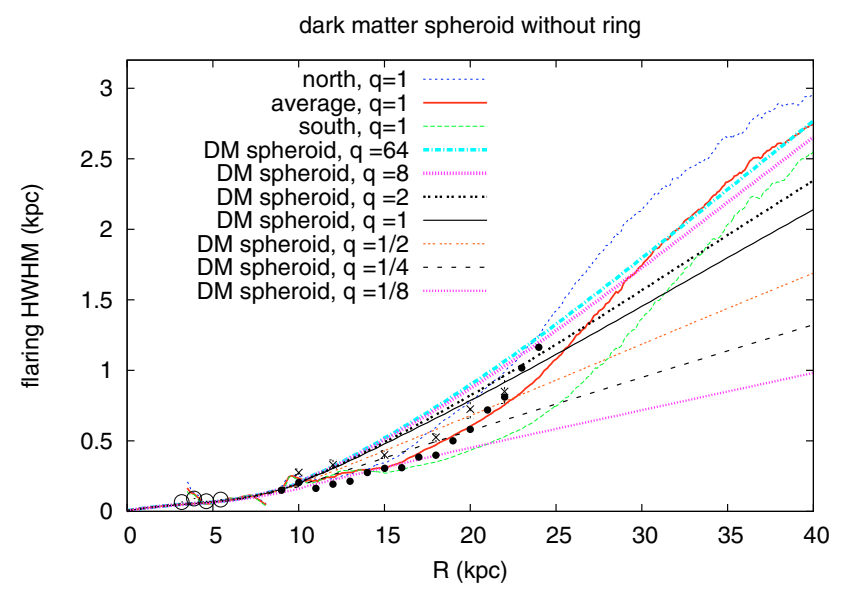

Fig. 4. Self-consistent H I flaring for isothermal dark matter spheroids. The thick red line gives the mean extension of the $\mathrm{H}$ I layer that should be comparable to the solid black line, which represents the flaring curve expected for the model with $q=1$. In addition, we include flaring curves for oblate halos with axial ratios $c / a=1 / 2$ to $1 / 8$ and also for prolate halos with axial ratios $c / a=2 / 1$ to $64 / 1$.

used data in the range $8 \lesssim R \lesssim 24 \lesssim \mathrm{kpc}$ to find that isothermal spheroids do not fit the observed flaring data particularly well. Using only the flaring at $R=2 R_{\odot}$, we could conclude that the halo must be rather oblate, $q \sim 0.1$, but Fig. 4 shows that the halo model needs to become increasingly prolate with distance. This reinforces the conclusion of Narayan et al. (2005) that a halo described by a single constant $q$ parameter does not match the observations if one considers a broad range of galactocentric distances.

\subsubsection{Flaring caused by a dark matter disk}

We tested alternatives to standard spheroidal halos and found that it is much easier to match a model to the observations if one considers dark matter associated with the Galactic disk. An example for the derived flaring curves is given in Fig. 5, with what we expect from our initial dark matter disk model (DMD) (Paper I). For comparison we plot the mean flaring determined from the derived $n(R, z, \phi)$ distribution for the northern and southern parts of the Milky Way disk.

Comparing the flaring derived from observations with the model, we generally find reasonable agreement between the two, comparable to the highly prolate spheroidal mass model $(q \sim 8)$ from Fig. 4 but with a better match for $12<R<25 \mathrm{kpc}$. However, we still have significant S-shaped deviations. We investigated whether these deviations could be explained by reduction problems. Each of our attempts to explain the discrepancies with problems during the data processing failed. Even trying deliberately to bias the data reduction led to no significant changes for the resulting $\mathrm{H}$ I distribution. We conclude that the deviations between model and data are highly significant and not caused by a systematic bias.

The depression in the flaring curve at $12<R<25 \mathrm{kpc}$ has been seen before, see e.g. the recent papers by Nakanishi $\&$ Sofue (2003) and Levine et al. (2006a), but the effect shows up as most pronounced in Fig. 23 of Voskes (1999). A low flaring can be explained either by an extraordinary low velocity dispersion of the H I gas or by a local enhancement of the mass distribution. The turbulent velocity dispersion in the interstellar medium (ISM) appears to be independent of distance (Blitz \& Spergel 1991; Burton et al. 1992). We find therefore

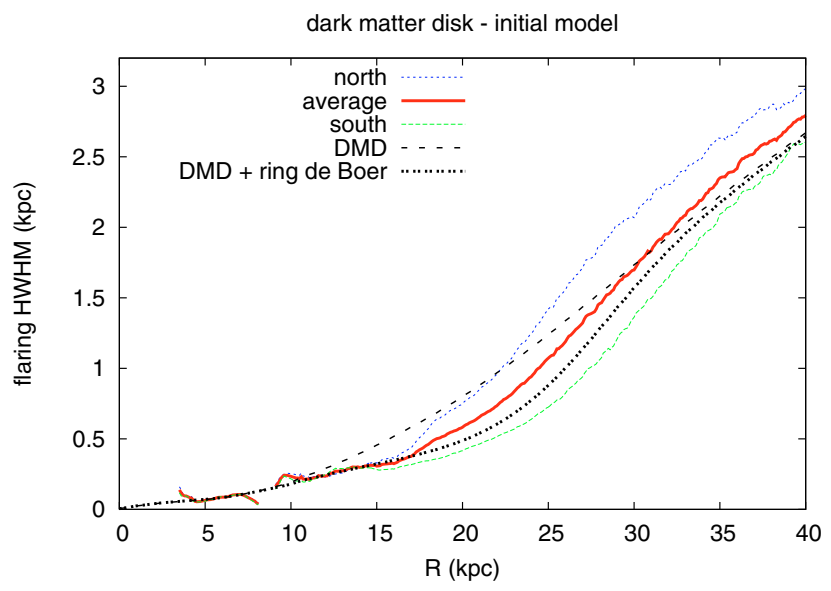

Fig. 5. Derived H I flaring for the initial dark matter disk model. We include the flaring curve as expected from the initial dark matter disk model (Paper I, black dashed) and after adding a dark matter ring to the model with parameters as given by de Boer et al. (2005) (black dotted).

no reason to assume that the $\mathrm{HI}$ gas is special at distances $15 \lesssim R \lesssim 20 \mathrm{kpc}$.

\subsubsection{Adding a dark matter ring}

None of the well-established dark matter models has an enhancement of the mass distribution at a distinct radial distance from the center, something our models seem to require to reduce or even eliminate the S-shape deviation shown in Fig. 5. However, a possible scenario could be a ring-like mass concentration caused by a merger event; see Hayashi et al. (2003) and Peñarrubia et al. (2005, 2006). Several groups claimed to have detected stellar streams in the Milky Way, right at the region of interest: Newberg et al. (2002); Ibata et al. (2003); Yanny et al. (2003); Martin et al. (2005); Martínez-Delgado et al. (2005); Bellazzini et al. (2006). Others explain the over-densities as due to the Galactic warp: Momany et al. (2004, 2006) and López-Corredoira (2006). The stellar mass of the giant stellar structure is estimated to be $2 \times 10^{8} M_{\odot}-10^{9} M_{\odot}$ (Ibata et al. 2003). It is, however, readily shown that such a mass is insufficient for explaining the low flaring at $R \sim 18 \mathrm{kpc}$.

A ring with a mass content well above the estimates for the stellar mass contribution is needed, and exactly this was recently claimed by de Boer et al. (2005) to exist. They studied the diffuse Galactic $\gamma$-ray background observed with EGRET. They find excess emission close to the Galactic disk and claim that the excess originates from ring-like dark matter distributions. One of the rings is located at $R \sim 14 \mathrm{kpc}$.

To test the de Boer et al. (2005) proposal, we include the ring at $R \sim 14 \mathrm{kpc}$ in our model (Fig. 5). The additional dark matter ring indeed provides a step in the right direction, but obviously the predicted flaring is now too low. We varied the position of the ring, the radial dispersion, and its total mass. All calculations included a self-consistent solution of the PoissonBoltzmann equation, a derivation of the Galactic velocity field, and finally a redetermination of the $3 \mathrm{D}$ volume density distribution $n(R, z, \phi)$ and the resulting flaring.

The best result is shown in Fig. 6. Note that not only the model flaring curve, but also the flaring derived from the data has changed in comparison to Figs. 3 to 5. The model reproduces the observations for a ring-like mass distribution

$n(R, z)=n_{r} \mathrm{e}^{-\left(R-R_{r}\right) /\left(2 \sigma_{r}^{2}\right)-\left|z / z_{r}\right|}$ 


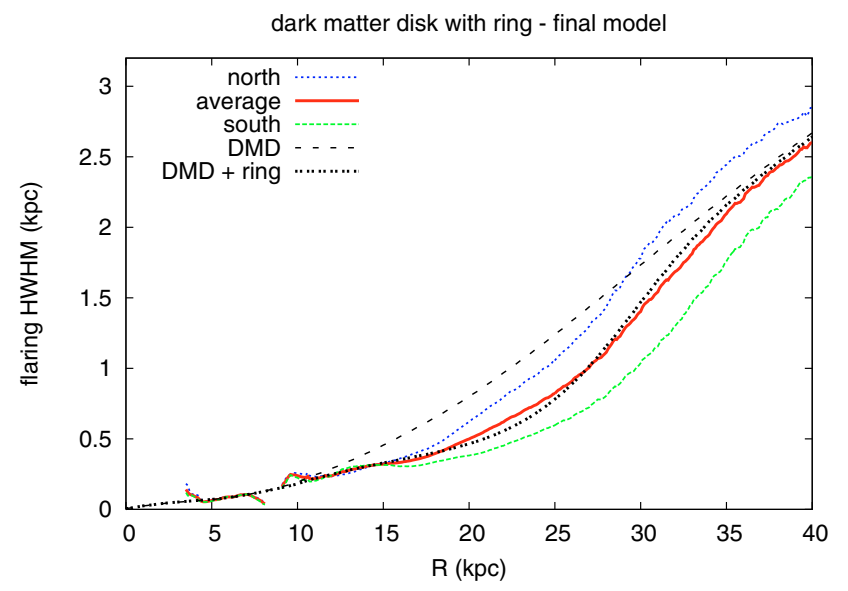

Fig. 6. Observed mean $H W H M$ flaring (thick red line), applying the rotation curve of our best-fit model. The dotted blue and green lines give the flaring in the northern and southern hemispheres, respectively. The best-fit flaring model, containing dark matter disk and ring, is plotted with a black dotted line. The flaring curve from the initial dark matter disk model (without ring) is shown for comparison (black dashed).

with $n_{r}=0.005 \mathrm{~cm}^{-3}, R_{r}=17.5 \mathrm{kpc}, \sigma_{r}=5 \mathrm{kpc}$, and $z_{r}=1.7 \mathrm{kpc}$. This relation was given by de Boer et al. (2005), but all parameters, except $z_{r}=1.7 \mathrm{kpc}$, have been redetermined and modified by us for an axisymmetric model. Discrepancies between our solution and parameters given by de Boer et al. (2005) may be explainable, at least partly, by uncertainties and systematical deviations of the ring-like mass distribution from axisymmetry. We determined here the mean properties of the ring alone. In Sects. 8 and 9 we discuss fluctuations in more detail. The method used by de Boer et al. (2005) is most sensitive in direction to the anti-center, a region which is seriously affected by velocity crowding, therefore not accessible to our analysis.

The resulting ring has a total mass of $2.3 \times 10^{10} M_{\odot}$. For the remaining part of the dark matter model we kept the model parameters as described in Table 1 of Paper I with only two changes. The local dark matter disk density had to be adjusted from $n=0.69 \mathrm{~cm}^{-3}$ to $n=0.75 \mathrm{~cm}^{-3}$, and we adopted a radial scale length $H_{R} / 2=3.75 \mathrm{kpc}$ for a better fit of the $\mathrm{H}$ I gas surface density.

\subsubsection{Spheroidal halo with dark matter ring}

Next we tested whether the inclusion of a dark matter ring can also lead spheroids to fit better. We consider three cases, $q=2$, 4 , and 64. Figure 7 shows that a prolate spheroid with $q=4$ represents a good fit, but observations cover the range $q=64$ for the northern and $q=2$ for the southern part of the Milky Way. The ring parameters used are identical to those discussed in Sect. 3.2.4, except that we need only $80 \%$ of the total ring mass in the case of a spheroidal halo. Solutions with different flattening parameters $q$ are nearly identical for $R \lesssim 25 \mathrm{kpc}$. This emphasizes once more the need to get constraints at large distances. Milky-Way mass models are ambiguous if a limited data base is used, a point stressed previously by Dehnen \& Binney (1998).

Comparing Figs. 7 with 6 formally results in an acceptable solution for $q=4$. Further improvements to the model (e.g. at $R \sim 15 \mathrm{kpc}$ and $R \sim 22 \mathrm{kpc}$ ) would be possible by splitting the dark matter ring into several lumps. We did not explore them for two reasons. i) Strongly prolate spheroids are rather unexpected from cold dark matter simulations (e.g. Dubinski \& Carlberg 1991); and ii) the surface densities at $R \sim R_{\odot}$ are

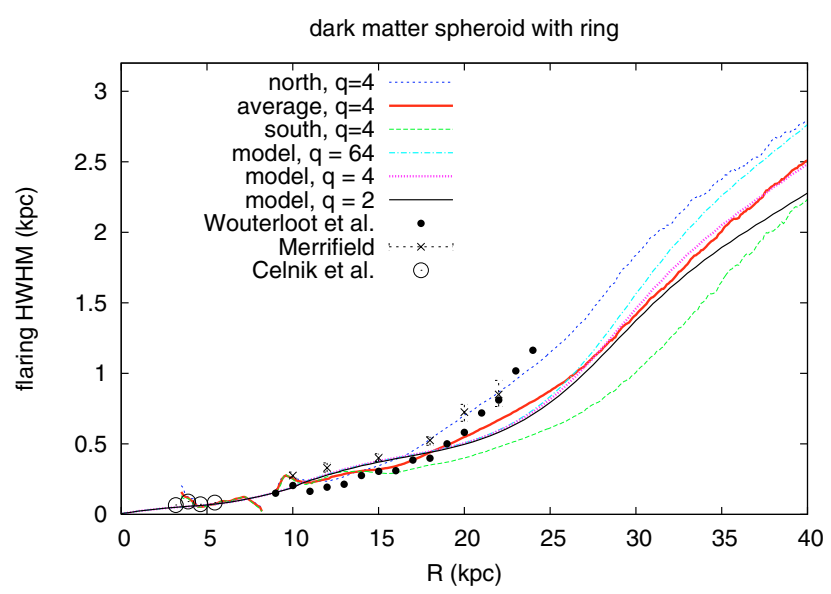

Fig. 7. Derived H I flaring for an isothermal dark matter spheroid with a dark matter ring. The symbols are the same as in Fig. 6. For comparison, flaring models are given for $q=2,4$, and 64 .

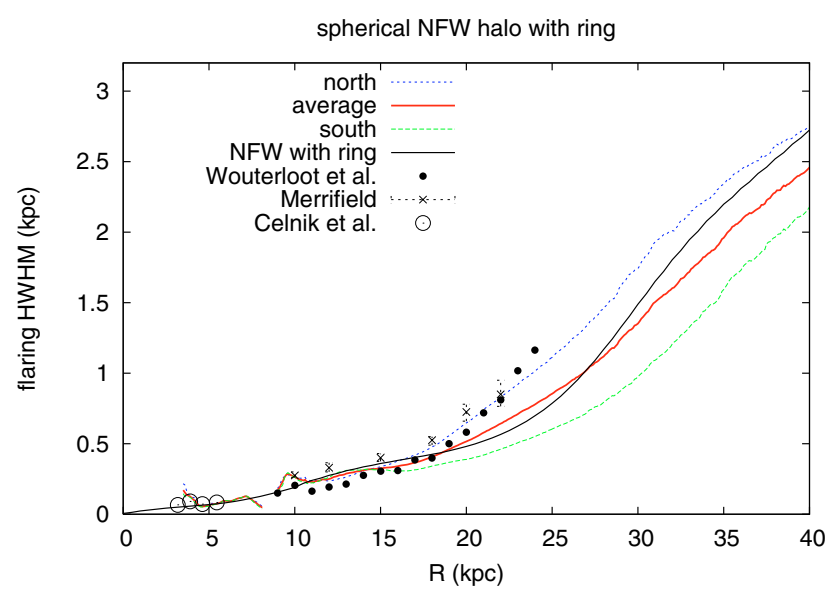

Fig. 8. Derived H I flaring for the NFW model. The solid black line gives the model. See Fig. 7 for further explanation.

unacceptable for all of our spheroidal models. In particular, highly prolate spheroids show the most significant deviations from HIPPARCOS results, see to Sect. 6 for further discussion.

\subsubsection{NFW halo with ring}

We also tried to fit a $\Lambda$ CDM-based NFW model (Navarro et al. 1996), using parameters provided by Klypin et al. (2002). The result is shown in Fig. 8 and fits the observations as well as the results presented in Figs. 6 and 7 and might be improved by assuming a lumpy ring structure. For a favored halo concentration parameter $C=12$, we needed, however, to modify the central mass density to keep $v_{\text {rot }}=220 \mathrm{~km} \mathrm{~s}^{-1}$, resulting in a total halo mass of $5 \times 10^{11} M_{\odot}$. This is in clear conflict with our constraint that the total mass of the Milky Way within $R \lesssim 350 \mathrm{kpc}$ should be close to $2 \times 10^{12} M_{\odot}$. Moreover, the halo mass is only $50 \%$ of the solution demanded by Klypin et al. (2002), likewise in conflict with the $\Lambda$ CDM constraints and therefore not acceptable. We were unable to match the flaring at large distances exactly with the observations (Fig. 8). It would be necessary to increase the halo mass, but this would imply at the same time that the local circular speed must significantly exceed $v_{\text {rot }}=220 \mathrm{~km} \mathrm{~s}^{-1}$. For the NFW model considered, there is too much mass in the inner part of the Galaxy. This is the well-known cusp problem. 


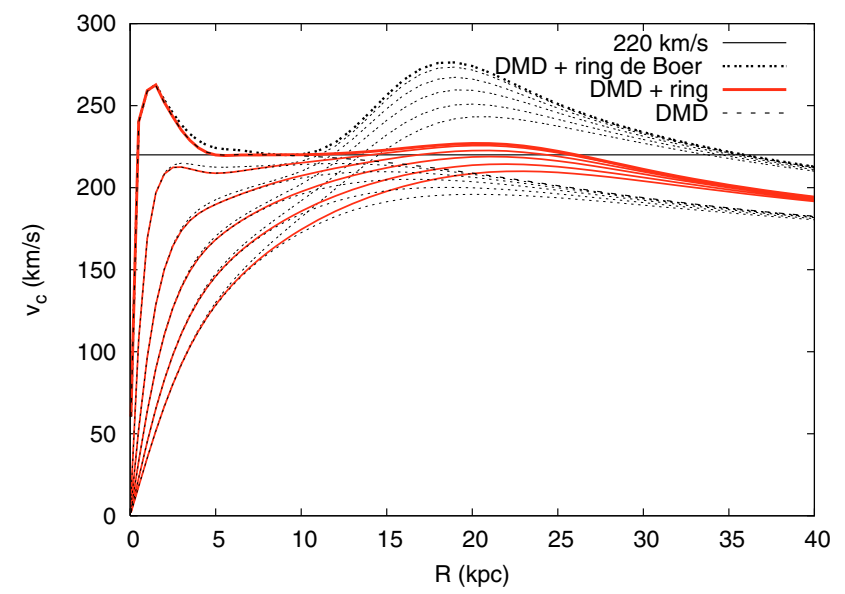

Fig. 9. Rotation curves for the models displayed in Figs. 5 and 6. The black dashed lines give the rotation curves for the initial dark matter disk, and the dotted lines the curves for the dark matter disk including a ring according to de Boer et al. (2005). The thick red lines show the rotation curve for the best-fit model. To the rotation at $z=0 \mathrm{kpc}$ (top thick curves) we add in each case the circular streaming velocities at $|z|=1$ to $5 \mathrm{kpc}$ (from top to bottom).

\section{Galactic rotation}

Each of the mass distributions discussed in the previous section causes a different rotation curve. This was taken into account when we derived self-consistent flaring curves for these models. Here we restrict the discussion to the dark matter disk model.

The 3D rotation curves for this model are shown in Fig. 9. We plot the initial dark matter disk, the proposal by de Boer et al. (2005), and the final best-fit. In each case we show the circular streaming velocities at $|z|=0$ to $5 \mathrm{kpc}$ (from top to bottom). Including a dark matter ring in our model causes the initially falling rotation curve of the dark matter disk (DMD) model to rise significantly at $R \sim 18 \mathrm{kpc}$. Adjusting the mass density of the ring to match the $\mathrm{H}$ I flaring brings the rotation curve down. Our best-fit mass model leads to a surprisingly simple result. The rotation law at $z=0 \mathrm{kpc}$ is almost flat over a range $5 \lesssim R \lesssim 27 \mathrm{kpc}$.

A flat rotation curve appears to conflict with observations (e.g. Blitz 1979; Schneider \& Terzian 1983; Fich et al. 1989; Merrifield 1992; Brand \& Blitz 1993; Honma \& Sofue 1997) which instead result in the high velocities of the de Boer et al. (2005) model. However, the discrepancy can be solved immediately if one takes distance uncertainties into account. The tracers used by various authors to measure the Galactic rotation curve for $R \gtrsim 11 \mathrm{kpc}$ have large distance errors. Binney \& Dehnen (1997) assumed that most of these objects are located within a ring at $R \sim 14 \mathrm{kpc}$ and derived for this case a flat rotation curve.

\subsection{The co-rotation problem}

In Fig. 10 we display the volume density distribution at a constant galactocentric distance $R=10 \mathrm{kpc}$, derived for the standard model with a constant rotation velocity $v_{\text {rot }}=220 \mathrm{~km} \mathrm{~s}^{-1}$. Figure 11 displays the distribution at the same distance, but for our best-fit model. Discrepancies are obvious, because they are caused by the different rotation curves. The velocities at $z=$ $0 \mathrm{kpc}$ are identical for both cases (see Fig. 9). Correspondingly, the densities at $z=0 \mathrm{kpc}$ are the same. The standard model (Fig. 10) has a cylindrical rotation, but for the best-fit model

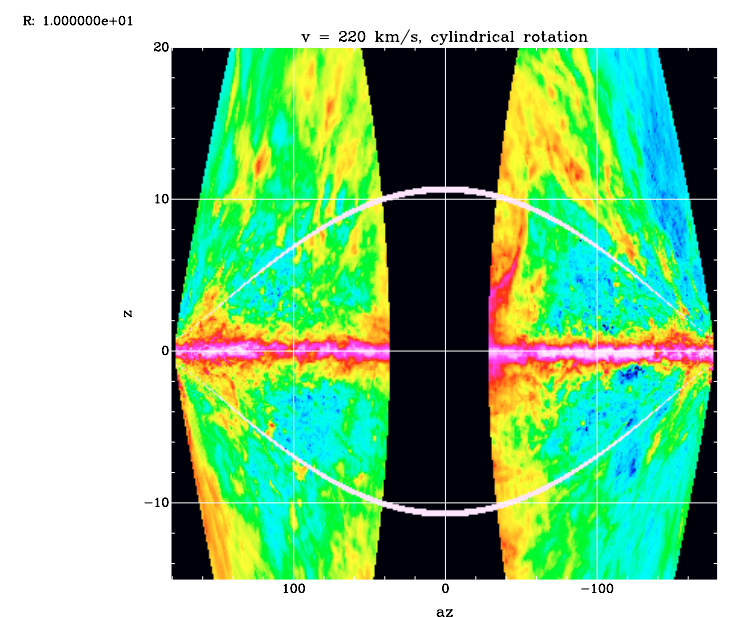

Fig. 10. Volume density distribution of the standard model, assuming cylindrical rotation, derived at $R=10 \mathrm{kpc}$ for azimuth $180^{\circ}>\phi>$ $-180^{\circ}$ and $-15<z<20 \mathrm{kpc}$. Densities affected by local emission with $\left|v_{\mathrm{LSR}}\right|<10 \mathrm{~km} \mathrm{~s}^{-1}$ are flagged dark. The white lines indicate latitudes $|b|=30^{\circ} \pm 0.5^{\circ}$. Only densities below $n<0.5 \mathrm{~cm}^{-3}$ are displayed. We use the same logarithmic transfer function as in Figs. 1 and 2.

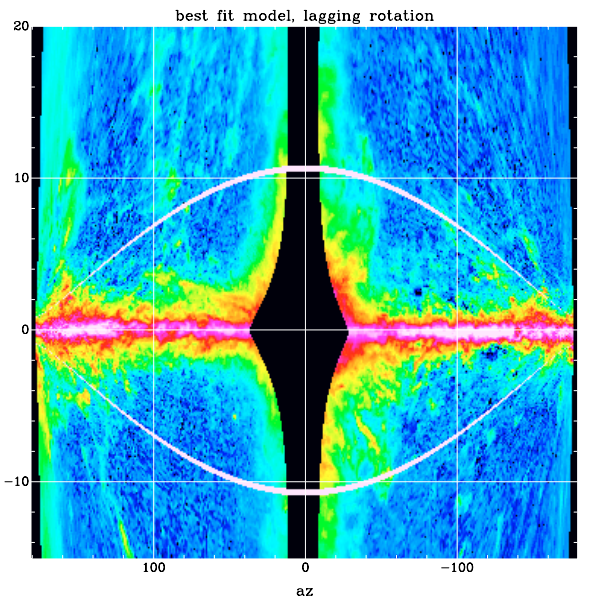

Fig. 11. Volume density distribution derived at $R=10 \mathrm{kpc}$ for the bestfit model with a lagging rotation according Eq. (1). For more details see Fig. 10.

(Fig. 11) $v_{\text {rot }}(R, z)$ lags according to Eq. (1) for increasing $z$ distances increasingly behind the disk.

We emphasize that Figs. 10 and 11 have been derived by running identical procedures, the only difference being the $3 \mathrm{D}$ velocity field. Comparing distinct features off the plane in the $3 \mathrm{D}$ volume density distribution $n(R, z, \phi)$ for both rotation laws, we find that structures, most noticeabley cloud complexes, are shifted to larger $R$ and $|z|$ distances if one assumes a cylindrical rotation. An example, the HVC complex R, was discussed in Sect. 3.1.

The average $\mathrm{H}$ I volume density of the disk $\langle n(R, z)\rangle$ falls off exponentially with radius $R$ and height $|z|$. Shifting distinct features to larger $R$ and $|z|$ distances therefore leads on average to significantly higher densities for $|z|>0 \mathrm{kpc}$. This effect is obvious if one compares the volume density distribution at a constant distance $R$ as derived for cylindrical rotation with that derived from a lagging rotation model. The flaring is larger if one assumes cylindrical rotation (Figs. 3 and 6). Discrepancies are 


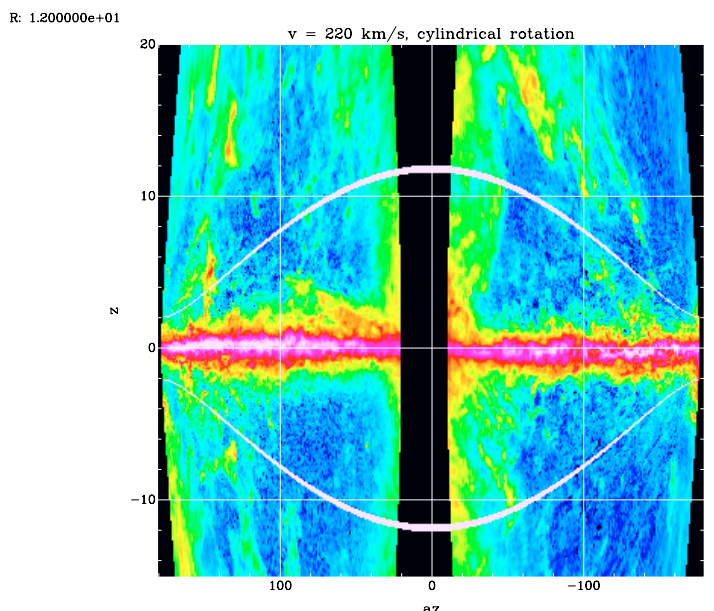

Fig. 12. Volume density distribution derived for $R=12 \mathrm{kpc}$ for the standard model with cylindrical rotation.

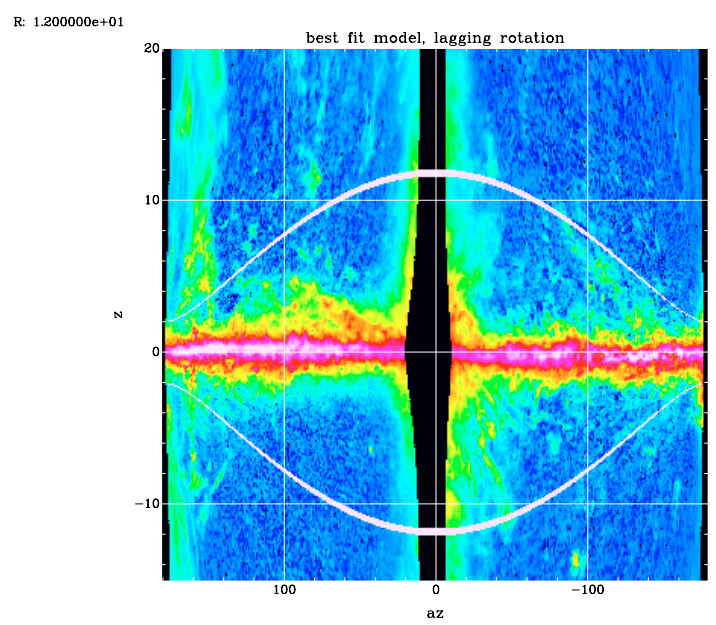

Fig. 13. Volume density distribution derived for $R=12 \mathrm{kpc}$ for the best-fit model with a lagging rotation law.

most significant close to the solar circle, but Figs. 12 and 13 derived at $R=12 \mathrm{kpc}$ demonstrate that even larger distances may be affected.

For our analysis we chose the lagging rotation according to Eq. (1) since it considerably improves the internal consistency of our results in comparison to a cylindrical rotation. We neglect buoyancy and any dependency of gas pressure terms $\nabla p / \rho$ on radius (Kalberla 2003, Sect. 5.5.1). These second-order corrections, discussed also by Benjamin (2002), may get important for extra-planar gas layers but are not expected to be significant for the disk gas. For example, at $R=20 \mathrm{kpc}$, we expect a $10 \%$ effect from the $\nabla p / \rho$ term only for $z \gtrsim 14 \mathrm{kpc}$. This $z$ distance is an order of magnitude off from the scale height of the disk gas so we ignore this correction for computational reasons.

Our preference for a lagging rotation with increasing $z$ distance is supported by observations that probe extra-planar gas, located on top of the standard disk gas we consider here. The average rotational streaming velocity in the Milky Way, as derived from interstellar absorption lines at $z$ distances up to a few kpc, is found to lag behind the disk (de Boer \& Savage 1983; Savage et al. 1997). Rand $(1997,2000)$ and Heald et al. (2006a,b) find evidence of lagging ionized gas in the halos of NGC 891 and NGC 5775. Very sensitive H I maps of NGC 891 (Swaters et al. 1997; Fraternali et al. 2005) and NGC 2403

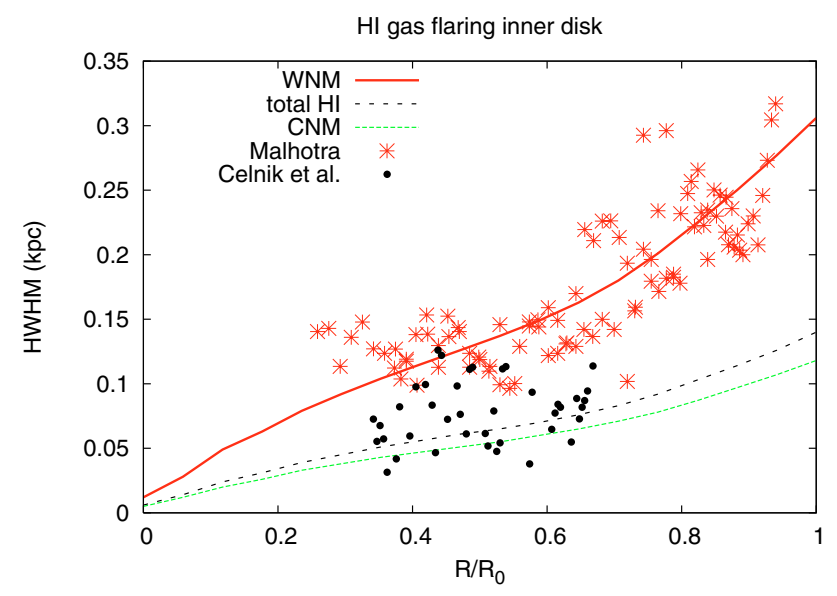

Fig. 14. Flaring of the H I gas in the inner Galaxy. The data, Malhotra (1995), (*), and Celnik et al. (1979), (•), represent the observed HWHM of H I clouds. The dashed black line represents the $H W H M$ as derived for a multiphase $\mathrm{H}$ I medium, the green dashed line is for the CNM, and the red solid line represents the WNM.

(Fraternali et al. 2001, 2004) similarly support a lagging extraplanar H I gas phase.

\section{Consistency check - available flaring data}

\subsection{Gaseous flaring inside the solar circle}

Our approach does not allow an accurate determination of the flaring inside the Solar circle, but we may compare our derived flaring with more accurate data. Figure 14 shows the flaring for the H I gas derived by Celnik et al. (1979) (•) and by Malhotra $(1995)(*)$. The results from these groups are found to be largely discrepant.

The scale height, derived by us from the model, is the scale height for a mix of the CNM and WNM. Both phases have significantly different scale heights. Celnik et al. (1979) have shown that the H I volume density distribution perpendicular to the Galactic plane can be described by a $\operatorname{sech}^{\alpha}\left(z / z_{0}\right)$ law. This relation can be approximated by a Gaussian distribution $\exp \left[-z^{2} /\left(2 z_{\mathrm{g}}^{2}\right)\right]$ close to the plane and at large distances by an exponential, $\exp \left(-z / z_{\mathrm{e}}\right)$. Bahcall (1984) demonstrated that such an approximation also applies for a multicomponent model if the gravitational potential can be approximated by a single dominating mass component. This approximation remains valid in the case of a more general mass distribution (see Paper I, Sect. 5.2.1). Celnik et al. (1979) fitted a general $\operatorname{sech}^{\alpha}\left(z / z_{0}\right)$ volume density law, solving for $z_{\mathrm{g}}$ and $z_{\mathrm{e}}$. This is the most accurate and unbiased way to determine the H I scale height. The determination of the first moment by us, excluding H I gas extending to large $z$ distances, is also unbiased. The approach by Malhotra (1995) is quite different. She assumed that the H I layer can be fitted by a single Gaussian component, represented only by the WNM (Fig. 14). Taking the different approaches into account, the flaring of the H I gas within the Solar circle is consistent with our model.

\subsection{Molecular gas}

In Fig. 15 we compare scale heights for the molecular gas phase from the best-fit dark matter disk model with observed scale heights. In the inner Galaxy two independent analyses are available from Bronfman et al. (1988) and Malhotra (1994). Both 


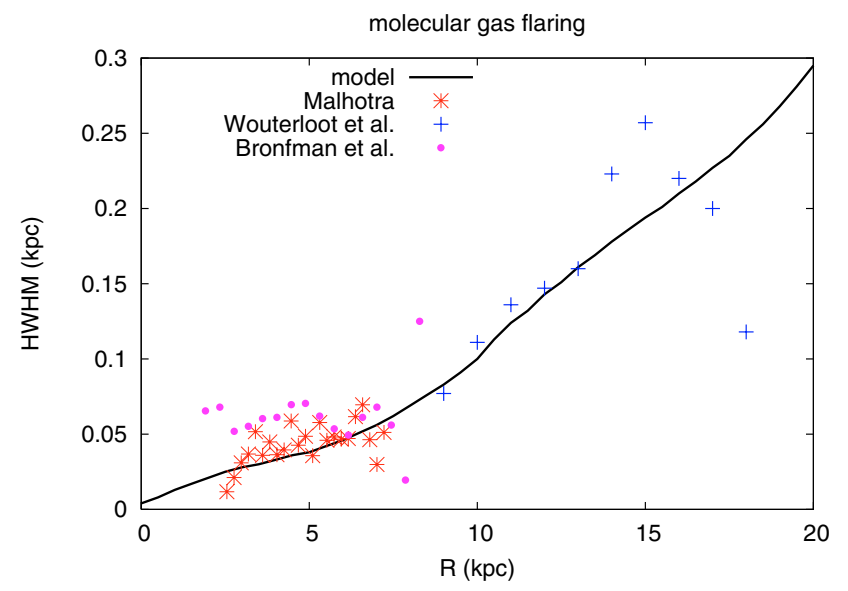

Fig. 15. Flaring of the molecular disk. The solid line represents the flaring for the final model. The data points give $\mathrm{CO}$ scale heights as derived by Malhotra (1994), (*), Wouterloot et al. (1990), (+) and Bronfman et al. (1988), (•).

sets of values appear to be slightly offset from each other. This may be explainable by details of the data analysis but is certainly unaffected by complications from a multi-phase composition as discussed in the previous section. For the outer Galaxy we used scale heights as derived by Wouterloot et al. (1990) for a verification of the model. In general we find good agreement between model and data except for the outermost data points. At distances $R \gtrsim 15 \mathrm{kpc}$, the observations may be limited in sensitivity.

\section{Consistency check - local mass surface densities}

Among the various observational constraints that may be used for determining the dark matter properties, the surface density $\Sigma_{1.1}$ within $1.1 \mathrm{kpc}$ is the measure with the lowest relative uncertainties (Kuijken \& Gilmore 1991). It therefore provides one of the most important constraints for models. Recent surface-density determinations based on HIPPARCOS have added further constraints.

Kuijken \& Gilmore (1991) determined $\Sigma_{1.1}=(71 \pm$ 6) $M_{\odot} \mathrm{pc}^{-2}$ using $\mathrm{K}$ dwarfs. Holmberg \& Flynn (2004) analyzed HIPPARCOS K giants and find $\Sigma_{1.1}=(74 \pm 6) M_{\odot} \mathrm{pc}^{-2}$. Our best-fit model provides a consistent solution for $\Sigma_{1.1}=$ $(79 \pm 2) M_{\odot} \mathrm{pc}^{-2}$. Within $800 \mathrm{pc}$ Holmberg \& Flynn (2004) determine a surface density of $\Sigma_{8}=(65 \pm 6) M_{\odot} \mathrm{pc}^{-2}$, we get $\Sigma_{.8}=(66.9 \pm 2) M_{\odot} \mathrm{pc}^{-2}$. For distances up to $350 \mathrm{pc}$, Holmberg \& Flynn (2004) determine $\Sigma_{.35}=41 M_{\odot} \mathrm{pc}^{-2}$, and Korchagin et al. (2003) find $\Sigma_{.35}=(42 \pm 6) M_{\odot} \mathrm{pc}^{-2}$. Our result is $\Sigma_{.35}=(42.7 \pm 2) M_{\odot} \mathrm{pc}^{-2}$.

For $z=0 \mathrm{pc}$, we find an Oort limit of $\rho_{\odot}=0.097 M_{\odot} \mathrm{pc}^{-3}$ in excellent agreement with Holmberg \& Flynn (2000), who obtained the same value, and only marginally deviating from $\rho_{\odot}=0.10 M_{\odot} \mathrm{pc}^{-3}$ derived by Kuijken \& Gilmore (1989) and $\rho_{\odot}=(0.105 \pm 0.005) M_{\odot} \mathrm{pc}^{-3}$ by Korchagin et al. (2003). The total surface density for all disk material (stars and gas) in our best-fit model is $52.5 M_{\odot} \mathrm{pc}^{-2}$, almost identical with the model estimate $52.8 M_{\odot} \mathrm{pc}^{-2}$ by Holmberg \& Flynn (2004). Summarizing, the local volume and surface densities are in excellent agreement with constraints by Kuijken \& Gilmore (1991) and with all constraints derived from HIPPARCOS. The uncertainties given for our model are based on typical parameter changes corresponding to modifications of the ring parameters

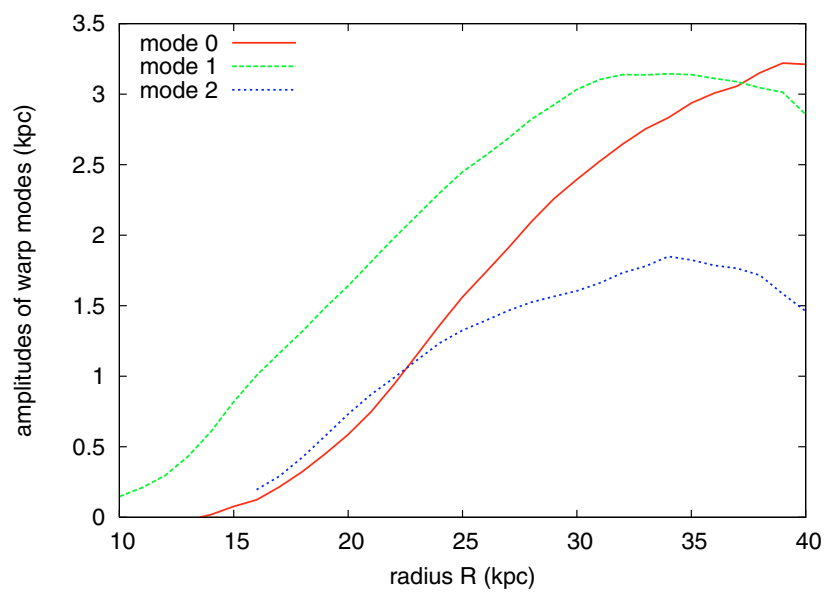

Fig. 16. Amplitudes for the warp modes 0 to 2 derived for the best-fit model. Mode 2 is insignificant for $R<15.5 \mathrm{kpc}$.

within an acceptable range. Our initial dark matter disk model without ring had $\Sigma_{1.1}=72.5 M_{\odot} \mathrm{pc}^{-2}$, but adding the dark matter ring parameters as initially proposed by de Boer et al. (2005) resulted in $\Sigma_{1.1}=88.3 M_{\odot} \mathrm{pc}^{-2}$, too high in comparison to the HIPPARCOS result of $\Sigma_{1.1}=(74 \pm 6) M_{\odot} \mathrm{pc}^{-2}$. This model got acceptable only after tuning with respect to the observed $\mathrm{H}$ I flaring (Sect. 3.2.4).

All of the conventional halo-mass models, discussed in Sects. 3.2.2, 3.2.5 and 3.2.6 have surface densities $\Sigma_{1.1}$ that deviate by at least $30 \%$ from $\Sigma_{1.1}=(74 \pm 6) M_{\odot} \mathrm{pc}^{-2}$, derived from HIPPARCOS (Holmberg \& Flynn 2004). Extreme prolate spheroids (Figs. 4 and 7) show the worst deviations.

\section{The Galactic warp}

It is well known since Westerhout (1957) that the Milky Way $\mathrm{H} \mathrm{I}$ disk is warped. We need to take this into account. The gravitational potential for our model is calculated for a flat system, but we apply a geometrical transformation afterwards to warp the system. We describe $W(R, \phi)$ as the deviation of the center of the gas layer from $z=0$ by

$$
\begin{aligned}
W(R, \phi)= & W_{0}(R)+W_{1}(R) \sin \left(\phi-\phi_{1}(R)\right) \\
& +W_{2}(R) \sin \left(2 \phi-\phi_{2}(R)\right),
\end{aligned}
$$

where $W_{i}$ and $\phi_{i}$ the amplitudes and phases of mode $i$.

To derive the warp we averaged the observed densities within rings having a width of $\Delta R=1 \mathrm{kpc}$ for $R=10$ to $40 \mathrm{kpc}$. For each annulus we fit the modes 0 to 2 simultaneously using a least square sine wave fit based on the Marquard/Levenberg algorithm (Press et al. 1986). Mode 2 is found to be insignificant for $R<$ $16 \mathrm{kpc}$. The fit in this range is therefore without mode 2 . We found that azimuth angles $80^{\circ} \lesssim \phi \lesssim 130^{\circ}$ for $R \gtrsim 16 \mathrm{kpc}$ show significant perturbations and exclude this range (region $\mathrm{X}$ as discussed by Levine et al. 2006a).

The formal errors for our fitted parameters are quite low, a few percent for the amplitudes, and typically less than a degree for the phases. Only at large distances do the errors increase for mode 2 . At $R \sim 40 \mathrm{kpc}$ we find a $10 \%$ amplitude error, the typical mode 2 phase error is $3^{\circ}$.

Figure 16 displays the amplitudes for the basic warp modes. We find a good agreement with Levine et al. (2006a). In Fig. 17 we display the warp phases. We followed the convention used by Levine et al. (2006a) and plot the phases for the first maximum close to azimuth $90^{\circ}$. Here we find some differences for 


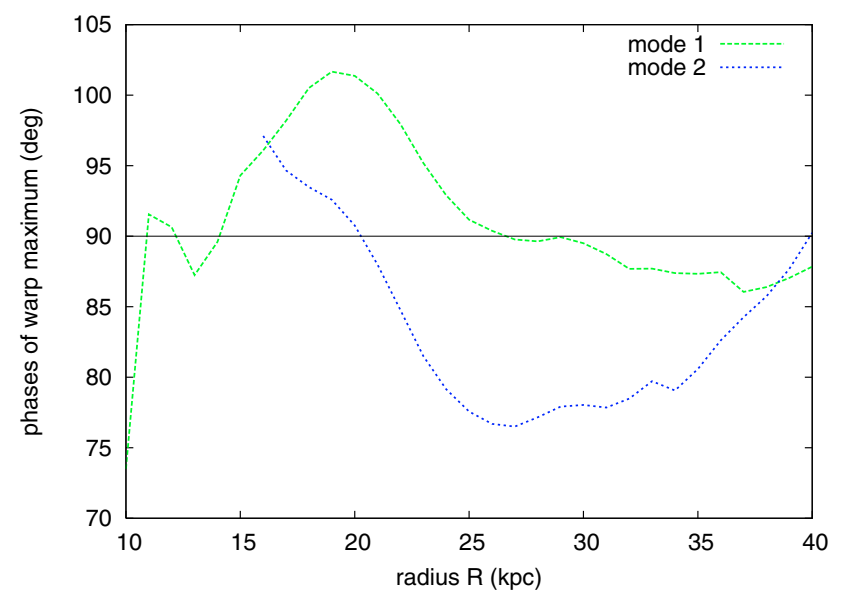

Fig. 17. Phases for the warp modes 1 and 2 derived for the best-fit model. Mode 2 is insignificant for $R<15.5 \mathrm{kpc}$.

the phase of mode 2, most probably due to the different rotation curve used by us. In Fig. 17 we see a well-defined maximum in the phase of mode 1 between $15 \lesssim R \lesssim 25 \mathrm{kpc}$. This appears to be associated with an almost constant slope in the phase of mode 2. Formal errors for the phases in this range are very low, less than one degree.

The warp modes are well-defined. The amplitude of mode 0 rises continuously up to $R \sim 40 \mathrm{kpc}$. Modes 1 and 2 both show a comparable trend in their amplitudes, peaking close to $33 \mathrm{kpc}$. The phases are also quite regular.

\section{Deviations from axisymmetry}

We modeled the flaring of the Milky Way H I gas assuming that the distribution of mass and gas in the Milky Way is in equilibrium. An axisymmetric model was used to fit the mean flaring. Figures 3 to 8 indicate systematic differences for azimuth angles $\phi \lesssim 180^{\circ}$ (northern sky) and $\phi \gtrsim 180^{\circ}$ (southern sky), and all flaring curves show in general the same trends: The axisymmetric model does not reproduce the flaring exactly but represents average properties of the mass distribution, implying a massive exponential disk with an additional massive ring at $R \sim 17.5 \mathrm{kpc}$. Here, we intend to discuss systematical perturbations from axisymmetry.

\subsection{A lopsided disk}

We calculated the average flaring within $20^{\circ}$ wide sectors. Figure 18 shows for the southern part of the Galactic disk that the sector averages are well-defined, deviating only a few percent from the mean. In the northern part (Fig. 19) the situation is very different. For radial distances $R \gtrsim 25 \mathrm{kpc}$ and azimuth $50^{\circ} \lesssim \phi \lesssim 130^{\circ}$, we find a significant increase in the flaring. The strongest deviation from the average flaring is at $90^{\circ} \lesssim \phi \lesssim 110^{\circ}$. It has already been noted by Levine et al. (2006a) that this region is special. Inspecting the survey data, we find a highly disrupted $\mathrm{H}$ I distribution with some evidence that the $\mathrm{H} \mathrm{I}$ in this range may be significantly perturbed (see Figs. 1 and 2). We exclude this sector from the average over the rest of the disk.

Systematic deviations in the flaring between northern and southern part of the disk are explainable if either the mass distribution is non-axisymmetric or the state of the ISM is deviant for a part of the disk. Explaining the asymmetries for $R \gtrsim 25 \mathrm{kpc}$ as merely due to the mass distribution leads to a dark matter

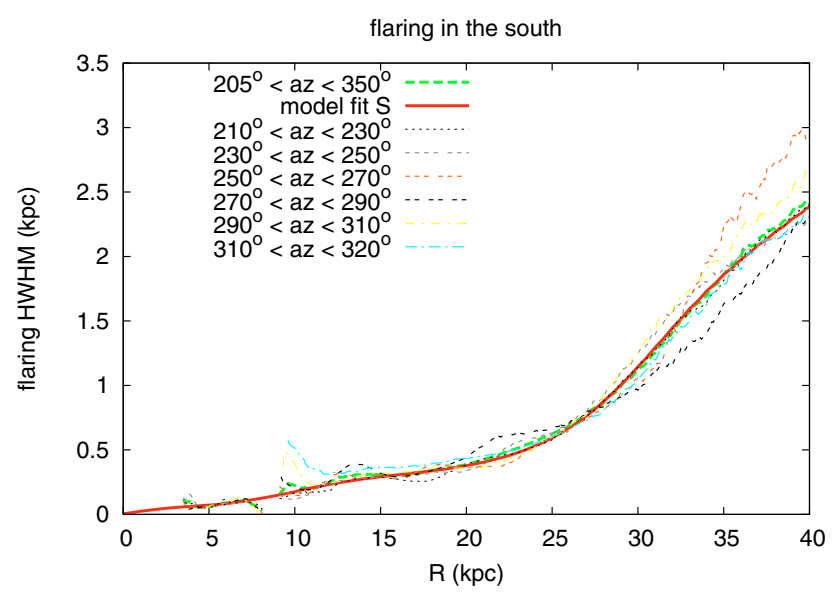

Fig. 18. Flaring of the southern part of the Galactic disk. The average flaring for $205^{\circ}<\phi<350^{\circ}$ (dashed green) is compared with the model fit (thick red). Flaring curves, derived for individual $20^{\circ}$ wide sectors, show good internal consistency of the flaring for the southern part of the disk.

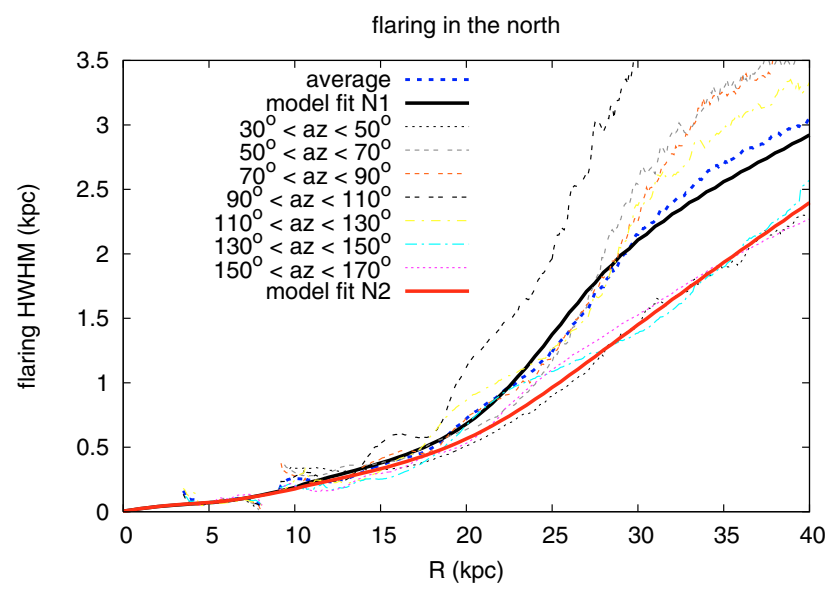

Fig. 19. Flaring of the northern part of the Galactic disk. The average flaring, excluding $50^{\circ}<\phi<130^{\circ}$, is indicated by the dashed blue line, the corresponding fit (N1) by the black line. Flaring curves derived for $20^{\circ}$ wide sectors show systematical deviations for $R>25 \mathrm{kpc}$. The thick red line shows the fit (N2) to the low flaring part of the H I, for $30^{\circ}<\phi<50^{\circ}$ and $130^{\circ}<\phi<170^{\circ}$.

model that in the southern part has three times the mass of the northern hemisphere (the fits S and N1 in Figs. 18 and 19). Correspondingly, the rotation velocities at $R \gtrsim 25 \mathrm{kpc}$ should vary by $50 \mathrm{~km} \mathrm{~s}^{-1}$. This is not observed so we exclude this possibility.

Taking the opposite point of view that the flaring is affected by variations in the state of the ISM, we assume for the moment that the flaring for $50^{\circ} \lesssim \phi \lesssim 130^{\circ}$ is "atypical" and may be disregarded (a more detailed discussion is given in Sect. 9). For the low flaring part of the northern disk we find that the dark matter disk has to be somewhat more extended (radial scale length $H_{R} / 2=8.75 \mathrm{kpc}$ ) than the disk fitted to the mean flaring used in the previous sections $\left(H_{R} / 2=7.5 \mathrm{kpc}\right)$. For the southern part of the disk $\left(205^{\circ}<\phi<350^{\circ}\right)$, we find almost identical parameters, $H_{R} / 2=8.5 \mathrm{kpc}$. Fitting the low flaring sectors only (model N2 $\& \mathrm{~S}$ ) results in a dark matter disk that is, within the errors, axisymmetric. The total mass of the dark matter disk is affected by the choice of the model. For best-fit model as displayed in Fig. 6 we obtain a mass $M_{\mathrm{DMD}}=1.8 \times 10^{11} M_{\odot}$. Fitting only the low 


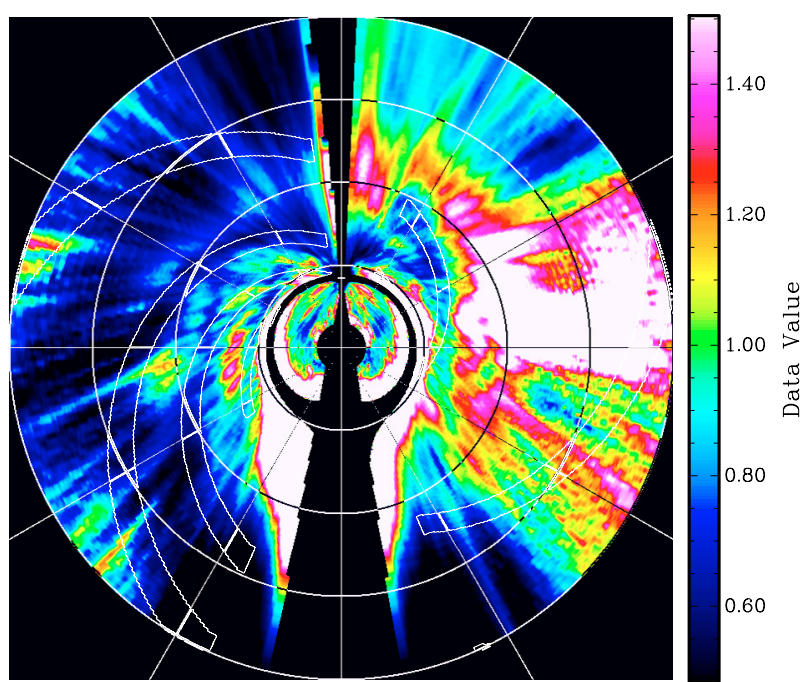

Fig. 20. Deviations $H W H M_{\text {obs }} / H W H M_{\text {model }}$ for the observed flaring relative to the best-fit model. The location of spiral arms according to Levine et al. (2006b) is indicated. The circles indicate galactocentric radii of 10, 20, 30, and $40 \mathrm{kpc}$.

flaring part of the H I disk (fits N2 \& S in Figs. 18 and 19) leads to $M_{\mathrm{DMD}}=2.4 \times 10^{11} M_{\odot}$.

Major north/south differences between the models remain and are easily visible in Figs. 18 and 19 at $R \sim 25 \mathrm{kpc}$. This is caused by variations in the dark matter ring radius with best-fit results of $R=13 \mathrm{kpc}$ in the north and $R=18.5 \mathrm{kpc}$ in the south. Similar variations between 15 and $20 \mathrm{kpc}$ for the observed stellar ring structure have been noted by Ibata et al. (2003). The total mass of the dark matter ring is $M=2.2$ to $2.8 \times 10^{10} M_{\odot}$. Fits N1 and $\mathrm{S}$ imply further that the southern part of the dark matter ring is three times as massive as the northern part. Our finding is consistent with known stellar over-densities in the south (e.g. Bellazzini et al. 2006), suggesting an association between dark matter ring and stellar distribution. The ring, however, cannot be associated with the Canis Major over-density. The radius $R=18.5 \mathrm{kpc}$ of the dark matter ring in this direction implies that this object is located behind Canis Major and instead associated with "the One Ring" discussed previously by Newberg et al. (2002); Ibata et al. (2003); Rocha-Pinto et al. (2003) and Yanny et al. (2003).

North/south differences in the mass distribution, considering the mass distribution from dark matter ring and disk alltogether (fits N2 \& S), lead to a lopsided dark matter distribution that is most significant at $R \sim 25 \mathrm{kpc}$. Our fits imply that the rotational velocities at this distance deviate by $\sim 15 \mathrm{~km} \mathrm{~s}^{-1}$, in good agreement with the empirical first-order epicyclic streamline correction derived by Levine et al. (2006a). We applied this correction as described in Sect. 2.1.

\subsection{Spiral structures}

For a better understanding of the nature of the large-scale fluctuations, we display in Fig. 20 the relative deviations of the observed flaring from the axisymmetric model defined as $H W H M_{\text {obs }} / H W H M_{\text {model }}$. The north-south asymmetry is obvious, but we also find ring-like perturbations and spiral arm features. Comparing Fig. 20 with perturbations in gas thickness as determined by Levine et al. (2006b, Fig. 2) shows excellent agreement, although very different methods have been used. This applies to the filtering of the data base for the separation of

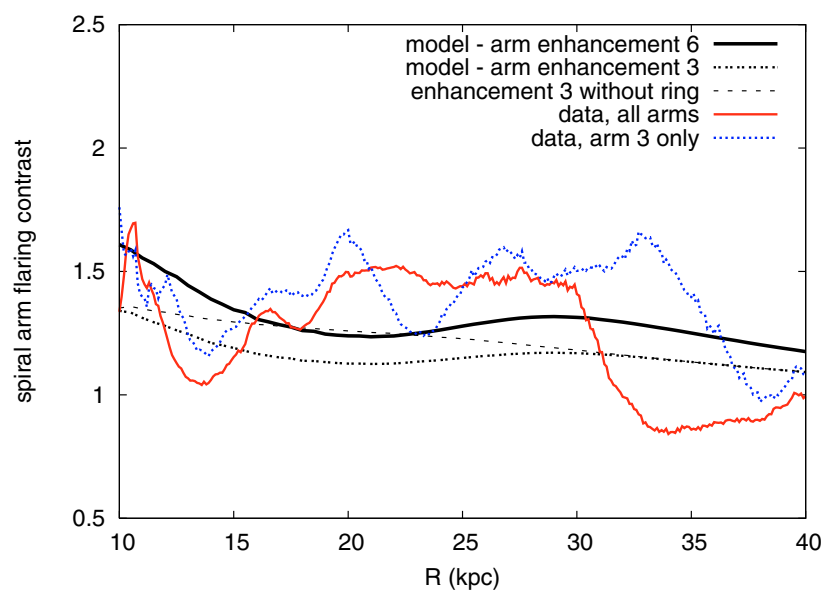

Fig. 21. Flaring contrast $H W H M_{\text {inter-arm }} / H W H M_{\text {arm }}$ between inter-arm and arm regions derived for the arms indicated in Fig. 20. The black lines represent model calculations assuming that the flaring is affected by gravitational forces from stellar spiral arms.

disk emission, to the determination of the scale heights, and in particular to the derivation of perturbations in scale height. Levine et al. (2006b) use the un-sharp masking method, while we compared the data with a model. The only agreement between both methods is that similar rotation curves were applied to converting the observed spectra to a $3 \mathrm{D}$ volume density distribution. Levine et al. (2006a,b) assume a flat rotation curve, $v=220 \mathrm{~km} \mathrm{~s}^{-1}$, while we derived the galactic rotation (Fig. 9) by fitting a mass model, also ending up with a flat rotation law for $z=0 \mathrm{kpc}$.

First of all we need to discuss the north-south asymmetry of the flaring. This begins at $R \gtrsim 15 \mathrm{kpc}$, about the same as the distance at which the warp causes a significant bending. The four-arm spiral pattern according to Levine et al. (2006b), indicated in Fig. 20, also shows a marked asymmetry with arms wide open to the north. Structures like these suggest tidal interaction. The Magellanic system might have caused a dark matter wake, enhancing the mass distribution towards the south (Weinberg 1998). The tidal interaction scenario is consistent with the onset of the warp and higher-mode vertical perturbations at larger distances (Saha \& Jog 2006; Levine et al. 2006a; Weinberg \& Blitz 2006). Large-scale deviations from axisymmetry, visible in Fig. 20, are fully consistent with such a scenario.

We used the spiral-arm parameters as determined by Levine et al. (2006b, Table 1) to verify whether the depressions in scale height along the spiral arms may be caused by gravitational forces from the stellar population. For the arm region we set a constant width of $\Delta \phi=15^{\circ}$ in azimuth, as indicated in Fig. 20. This is compared with an inter-arm region with a width of 17.5 on each side of the arm. Perturbations in direction to the Galactic center or anti-center, caused by spurious emission, are excluded as indicated. Figure 21 plots the ratio $H W H M_{\text {inter-arm }} / H W H M_{\text {arm }}$ determined as an average over all four arms and also for arm 3 alone. Arm 3 according to Levine et al. (2006b, Fig. 3) was chosen since it is particular well-defined. It runs from $R \sim 12 \mathrm{kpc}$, $\phi \sim 180^{\circ}$ to $R \sim 40 \mathrm{kpc}, \phi \sim 340^{\circ}$.

We used our model to calculate the flaring perturbations relative to an axisymmetric model, caused by enhancements of the stellar volume density within the arms by a factor of 3 or 6 over the axisymmetric model. A factor of 3 would be expected for well-defined spiral arms, as in M 51 (Rix \& Rieke 1993). A factor of 6 may be considered as an upper limit in the case of 
a four-arm spiral if the stars concentrate in arms with $\Delta \phi=15^{\circ}$ in azimuth.

Comparing the observed contrast with the model, we find that the observed amplitudes of the stellar spiral arms in the outer parts of the Milky Way are stronger than expected. This finding, however, is not solid enough to conclude that the spiral structure in the $\mathrm{H}$ I flaring must be caused by an additional mass component. Gas, according to our model, contributes only $13 \%$ to the baryonic surface density of the outer disk. The self gravity of the gas is therefore insignificant. The model parameters used for the stellar disk, in particular for the exponential radial scale length of the thick disk $\left(H_{R}=7.5 \mathrm{kpc}\right.$, Chiba \& Beers 2000), are highly uncertain. The surface densities for the outer stellar disk may be underestimated in our model. We conclude that spiral arms influence the mass distribution out to $R \sim 30 \mathrm{kpc}$, possibly even out to $R \sim 35 \mathrm{kpc}$ if we consider only arm 3 . For comparison, Levine et al. (2006b) find evidence of a spiral structure out to $R \sim 25 \mathrm{kpc}$. The presence of stellar spiral arms appears to be noticeable out to large distances, approximately $2 R_{25}$.

Figure 20 shows some ring-like fluctuations and Fig. 21 gives evidence of radial variations of the flaring contrast. A close inspection of Figs. 6, 18, and 19 also shows some oscillations if one compares the model flaring with the observations. Obviously the mean radius of the ring $(R \sim 17.5 \mathrm{kpc})$, as used in our model, is only a crude approximation of a more complicated pattern of radial substructures in the Milky-Way mass, as expected for merger events (e.g. Martin et al. 2005, Fig. 11). The tidal interaction scenario is consistent with the observed radial variations of spiral arm amplitudes that may arise from interference of a pre-existing spiral pattern with tidally-induced spiral arms (Rix \& Rieke 1993). Radial variations in the arm amplitudes in our Galaxy may be comparable to variations found in M 51. An alternative interpretation of radial fluctuations are ring-like mass concentrations caused by mergers. Figure 21 shows that these also influence the arm/inter-arm contrast.

In summary, we conclude that our model is able to represent the global properties of the Milky-Way mass distribution. Even substructures like spiral arms and radial fluctuations are explicable.

\section{Uncertainties}

In the previous sections we have claimed that a dark matter component associated with the Galactic disk can fit the observed $\mathrm{H}$ I flaring best, but at least we have found no easy way to match a standard model to the flaring data. The best conventional model, a highly prolate spheroid ( $2 \lessgtr q \lessgtr 64)$, appears unlikely since this is not what we expect from $\Lambda C D M$ models. Could it be that the analysis is systematically biased?

As discussed in the previous section, the gravitational field of the Milky Way must have significant non-axisymmetric contributions. Accordingly, the Galactic H I disk cannot be in an axisymmetric equilibrium state. In addition, stellar activities may lead to a fountain flow and mass may be accreted from outside (van Woerden et al. 2004). As part of our analysis we estimate the amount of gas in non-circular motion for each position in the Galactic plane, thus either representing out- or inflow. We exclude this gas from our analysis, on average 3\% to $5 \%$ of the total HI. The remaining uncertainties are most probably below a percent level, too low to bias derived flaring curves significantly.

Most critical for deriving halo properties is the modeling of the HI scale height. The conventional approach is to assume a single-phase medium with a velocity dispersion of $\sigma \sim 9.2 \mathrm{~km} \mathrm{~s}^{-1}$ (Olling \& Merrifield 2000, 2001; Narayan et al. 2005). We considered a more sophisticated two-phase medium with two different characteristic scale heights (Dickey \& Lockman 1990), defined by the turbulent motions within the H I gas. Using the Leiden/Dwingeloo Survey (Hartmann \& Burton 1997), Kalberla \& Kerp (1998) determined the average exponential scale height of the CNM to $150 \mathrm{pc}$ and the scale height of the WNM to $400 \mathrm{pc}$ (see also Table 1 in Paper I). The total scale height of the H I gas layer is in general given by the mix of both phases and therefore depends on the properties and the composition of the two phases throughout the disk.

The conditions for the existence of a two-phase medium have recently been discussed by Wolfire et al. (2003). These authors conclude that the CNM and WNM phases must coexist over most of the Galactic disk, at least out to radii of $16-18 \mathrm{kpc}$. Basically, such a two-phase medium can exist as long as the turbulent pressure of the WNM is high enough to drive parts of the H I gas into a cold dense phase. We repeated the calculations by Wolfire et al. (2003) of the parameters of the H I gas distribution. We find, for large radial distances, a 50\% higher turbulent pressure than used by Wolfire et al. (2003). This certainly supports a two-phase medium at large distances. From a Gaussian analysis of HVCs, Kalberla \& Haud (2006) find clear evidence of a two-phase medium in complex $\mathrm{H}(R \sim 30 \mathrm{kpc})$, the Magellanic stream, and the leading arm $(R \sim 50 \mathrm{kpc})$. In addition they argue for a high pressure to the hot halo gas phase. We conclude that there is strong evidence that the H I gas has a multiphase composition throughout most of the Milky Way disk. Any reservations that the CNM must be absent beyond the extent of the stellar disk (e.g. Olling \& Merrifield 2000, 2001) appear to be outdated.

The extended strongly flared region, visible in Fig. 20 at $R \gtrsim 25 \mathrm{kpc}$ and $50^{\circ} \lesssim \phi \lesssim 130^{\circ}$, deviates significantly from the average $\mathrm{H}$ I disk gas. This region has particularly low surface and volume densities and it is strongly warped. It is quite possible that the HI gas is out of equilibrium there and that the CNM phase may be under-abundant. Detailed investigations would be necessary to verify this, but they are clearly beyond the scope of this investigation.

Figure 14 displays scale heights of our model individually for the CNM and WNM phases, but also for the two-phase mix. Note that the scale height of the two-phase medium is only slightly larger than the scale height of the CNM. A WNM gas phase without any cold HI gas would result in a scale height that is twice as big as the scale height for the CNM/WNM mixture. This factor of two defines an upper limit for an excess in flaring caused by the transition of a multiphase gas into a WNM-dominated phase. Except for the range $90^{\circ} \lesssim \phi \lesssim 110^{\circ}$, such a factor applies to the region $R \gtrsim 25 \mathrm{kpc}$ and $50^{\circ} \lesssim \phi \lesssim$ $130^{\circ}$, described in more detail in Sect. 8.1. For the rest of the disk we have no indications that there are significant changes in the H I composition for $R \gtrsim R_{\odot}$ so use a constant column density ratio for the CNM and WNM phases throughout the Milky Way.

Calculating the scale heights from the mass model (Eq. (7) in Paper I), we are faced with the problem that individual gas components are not only supported by their own turbulence, but are also affected by the pressure of magnetic fields and cosmic rays. Following Parker (1966, 1969), the support can be described as an increase in the velocity dispersion by a factor $\sqrt{1+\alpha+\beta}$, where $\alpha$ stands for the increase due to the magnetic field and $\beta$ due to the cosmic-ray component. A simultaneous determination of the 3D distributions of gas, magnetic fields, and cosmic rays is needed to derive $\alpha$ and $\beta$. From observations, the distribution of H I, the diffuse ionized gas (Reynolds 1997), and the $10^{6.15} \mathrm{~K}$ plasma (Pietz et al. 1998), as well as the synchrotron radiation 
(Haslam et al. 1982) and the $\gamma$-ray emission (Fichtel et al. 1994), are available for this purpose. Based on a correlation of these datasets, Kalberla \& Kerp (1998) conclude that, for the CNM and the WNM gas phases, only a limited support is expected from a turbulent magnetic field component close to the disk with $\alpha \sim 1 / 3$. Accordingly, this leads to an effective increase in the velocity dispersion by a factor of $\sim 1.15$. For comparison, Olling \& Merrifield $(2000,2001)$, arguing that the pressure support can be derived from the scale heights of gas, estimate a value of $\sim 1.06$ for the increase in the effective velocity dispersion due to magnetic fields and cosmic rays.

Our algorithm allows using separate scale heights for the CNM and WNM, but we find no reason to vary the $\mathrm{CNM} / \mathrm{WNM}$ ratio or the pressure support from magnetic fields and cosmic rays with galactocentric radius. Our solution is close to a single-component model with an effective constant velocity dispersion of $8.3 \mathrm{~km} \mathrm{~s}^{-1}$, somewhat low in comparison to the preferred value of $9.2 \mathrm{~km} \mathrm{~s}^{-1}$ of Olling \& Merrifield (2000, 2001). These authors explored the parameter space, affecting $\mathrm{H}$ I flaring for various dispersions at a single constant galactocentric radius $R=2 R_{\odot}$. In contrast, we use a constant dispersion for a broad range of distances, and the question arises whether this is justified. It is very difficult to estimate how far the isothermal assumption may lead to possible biases. However, we may turn the question around and ask how far we would need to modify our ISM model assumptions if we want to save the paradigm of the Milky Way surrounded by a spheroidal halo.

For a spheroidal mass model without a ring and $q=1$ (Fig. 4) we would need to increase the estimate for the flaring at $R \sim 17 \mathrm{kpc}$ by $40 \%$ and, at the same time, to decrease the estimate at $R \sim 35 \mathrm{kpc}$ by $40 \%$. Pressure support from magnetic fields might be missing at large distances, which would bring the scale down by $15 \%$. In turn, we need to assume that velocity dispersion of the $\mathrm{H}$ I gas is overestimated by $25 \%$. Given the uncertainties, such a solution cannot be excluded. The arguments are the opposite at $R \sim 17 \mathrm{kpc}$. Here we need to boost the turbulent gas pressure by $40 \%$. However, the question immediately arises as to what kinds of processes would be available to feed this additional turbulence at distances $12 \lesssim R \lesssim 22 \mathrm{kpc}$. This region is largely outside the stellar disk, hence we expect no support from stellar activities. Figure 4 shows that highly prolate spheroids fit best at large distances. However, the problem remains to explain a turbulent pressure support at $R \lesssim 25 \mathrm{kpc}$. Alternatively, highly oblate models fit the observations well up to $R \lesssim 25 \mathrm{kpc}$, but it is then a serious problem to explain the observed flaring in the outskirts of the Milky Way.

Other mass models without dark matter in the Galactic disk might be considered. Narayan et al. (2005) advocate a model according to Eq. (2) with a power index $p=2$. Such a model fits the $\mathrm{H}$ I flaring well. The total halo mass is $2.8 \times 10^{11} M_{\odot}$, however, much too low to be acceptable. This model has most of its mass within $R \lesssim 50 \mathrm{kpc}$. Adding an extended halo to the Narayan et al. (2005) model would result in a bimodal dark matter model, very similar to our case.

\section{Summary}

The aim of our contribution is to derive an H I volume density distribution $n(R, Z, \phi)$ for the Milky Way. The main driver behind this is the availability of the LAB survey, a new all-sky H I survey (Kalberla et al. 2005). In particular, we expect that the low internal errors of this survey may lead to higher fidelity in the derived distribution.
Previously, this survey was used by Levine et al. (2006a,b), who assume a constant $v=220 \mathrm{~km} \mathrm{~s}^{-1}$ rotation law. In performing the $T_{B}(l, b, v)$ to $n(R, z, \phi)$ conversion we notice, however, that uncertainties in the Milky Way rotation curve are large enough to make the results ambiguous. We have tried to solve these problems by matching mass models of the Milky Way to the observations. The strategy is to obtain a self-consistent solution. We generated mass models that satisfy both the Poisson and the Boltzmann equation at the same time. To apply a model we require that predictions from the mass model need to agree with the derived volume density distribution. In particular, we used the global flaring of the H I gas layer to decide on the consistency of a model. The link between derived volume density distribution and flaring according to a mass model is the rotation curve, requiring a self-consistent mass distribution. Thus, our procedure is based on a closed loop.

\subsection{Properties of the mass model}

The Galaxy is embedded in an isothermal dark matter halo with a core radius of $35 \mathrm{kpc}$ and a total mass of $M=1.8 \times$ $10^{12} M_{\odot}$ within $350 \mathrm{kpc}$. To fit the global flaring of the H I disk, we need dark matter associated with the disk in the form of a self-gravitating exponential disk with a radial scale length of $7.5-8.75 \mathrm{kpc}$ and a mass $M_{\mathrm{DMD}}=1.8$ to $2.4 \times 10^{11} M_{\odot}$. To explain H I flaring at $13 \lesssim R \lesssim 20 \mathrm{kpc}$, we need an additional component. A dark matter ring at a radius $13 \lesssim R \lesssim 18.5 \mathrm{kpc}$ contributes $M=2.2$ to $2.8 \times 10^{10} M_{\odot}$. About $2 / 3$ of the ring mass is located in the south at $R \sim 18 \mathrm{kpc}$, while the remaining part of the ring mass is in the north at $R \sim 13 \mathrm{kpc}$.

According to our best-fit model (the average flaring as displayed in Fig. 6), the total mass of the Milky Way disk within $50 \mathrm{kpc}$ is $M=(2.9 \pm .1) \times 10^{11} M_{\odot}$. However, the state of the ISM at $R \gtrsim 22 \mathrm{kpc}$ is rather uncertain (Sects. 8 and 9). The northern part of the outer disk may be dominated by the WNM phase. Taking this into account by fitting the low flaring part of the $\mathrm{H}$ I disk only (fits N2 \& S in Figs. 18 and 19) leads to $M \sim 3.5 \times$ $10^{11} M_{\odot}$, probably an upper limit.

\subsection{Global properties of the HI disk}

Our best-fit model results in a rotation curve that is essentially flat for $R \lesssim 27 \mathrm{kpc}$ and falling off gradually for larger distances. Our derived H I volume density distribution resembles previous results obtained for a constant rotation (Voskes 1999; Levine et al. 2006a), but we derived cleaner pictures of the Milky Way $\mathrm{HI}$ gas distribution and extended the investigations to larger distances. A significant improvement is obtained by dropping the assumption that the gas is barotropic, hence in cylindrical rotation.

We derived a well-defined exponential H I disk with $n(r) \sim$ $\exp (-R / 3)$. The H I surface density distribution is also exponential and has a radial scale length of $3.75 \mathrm{kpc}$. The H I disk flares strongly. For $R \sim 4 \mathrm{kpc}$ the $H W H M$ scale height of the H I layer is $0.06 \mathrm{kpc}$, increasing to $2.7 \mathrm{kpc}$ at $\sim 40 \mathrm{kpc}$.

The H I disk shows systematic north-south asymmetries for $R \gtrsim 15 \mathrm{kpc}$, apparently correlated with the warp. The warp has well-defined Fourier modes 0 to 2 with slowly variable phases. The mean flaring in the northern part is up to a factor of 2 larger than the flaring in the south. 


\section{Discussion}

The dark matter disk, proposed by us, appears to conflict with results from HIPPARCOS (Holmberg \& Flynn 2000, 2004; Korchagin et al. 2003). The HIPPARCOS data are believed to imply that there is no evidence for significant amounts of dark matter in the disk. However, the flaw in this frequently used statement is that a definition of the disk is missing. Constraints from the stellar surface densities define only a local lower limit of 2-3 kpc for the scale height of a dark matter component associated with the disk. The dark matter disk in our model has a local scale height of $h_{z}=4 \mathrm{kpc}$ and matches all HIPPARCOS constraints. Models without any dark matter within the disk are not consistent with the observed gaseous flaring, in particular for distances $R \gtrsim 22 \mathrm{kpc}$.

A dark matter disk, or alternatively a highly prolate spheroid, explains the global flaring of the Galactic H I disk. But there remains a marked depression in the derived flaring at distances $15 \lesssim R \lesssim 20 \mathrm{kpc}$. To explain this it is in any case necessary to add a massive ring.

\subsection{Lord of the rings}

A longstanding discrepancy, discussed in Sect. 1, is that the observed rotational velocities for $R \gtrsim R_{\odot}$ conflict with mass models. To solve this problem, Binney \& Dehnen (1997) propose that most of the tracers that appear to be at $R \gtrsim 11 \mathrm{kpc}$ might actually be concentrated within a ring at $R \sim 14 \mathrm{kpc}$. Stellar over-densities suggesting a ring-like concentration were found a few years later by several groups independently. Perhaps the most concise description of this feature was given by Ibata et al. (2003); there is a giant stellar ring at $15 \lesssim R \lesssim 20 \mathrm{kpc}$ with a radial thickness of $2 \mathrm{kpc}$ and a vertical scale height of $\sim 0.75 \mathrm{kpc}$. The total stellar mass is estimated to be between $2 \times 10^{8} M_{\odot}$ and $10^{9} M_{\odot}$.

Such a mass, however, is insufficient for explaining the observed flaring. Independent evidence of a massive ring was given recently by de Boer et al. (2005). EGRET observations show excess in the diffuse Galactic $\gamma$-ray background. A possible explanation is that the giant stellar ring is associated with a dark matter ring. We included this mass component in our dark matter disk model but noticed that the mass of such a ring, initially proposed by de Boer et al. (2005), needs to be reduced, and the position and extension also needs to be slightly modified to explain the H I flaring. A fully satisfying self-consistent solution of our mass model and the average derived density distribution is obtained for a dark matter ring at $R \sim 17.5 \mathrm{kpc}$ with a radial extension of $5 \mathrm{kpc}$ and a mass $M=2.3 \times 10^{10} M_{\odot}$ (see Fig. 6). The associated rotation curve is almost flat out to $R \lesssim 27 \mathrm{kpc}$. This extended dark matter ring, associated with a somewhat less extended stellar ring and some enhancements in $\mathrm{HI}$ is completely consistent with the proposal by Binney \& Dehnen (1997) that distance errors may mimic a rising rotation curve for $R \gtrsim 11 \mathrm{kpc}$.

The de Boer et al. (2005) paper explains the EGRET excess emission as due to dark matter annihilation (DMA). From the energy spectrum of the excess radiation, they deduce a WIMP mass between 50 and $100 \mathrm{GeV}$. The significance of the EGRET excess emission was confirmed by Bergström et al. (2006), but these authors question that the excess can be caused by DMA. They model the decay processes proposed by de Boer et al. (2005) and derive that such a DMA would be associated by a significant antiproton flux. The expected flux is well above observational limitations, so they conclude that the signal cannot be caused by DMA. Bergström et al. (2006) propose to explain the
EGRET excess by enhancements in the baryonic matter distribution within the area covered by the ring.

As a crude order-of-magnitude estimate, the column density of the ring along the line of sight is comparable to the total column density of the observable H I disk in the same direction. For such baryonic matter contained within the usual gas phases, a huge EGRET signal would be expected. This is clearly incompatible with the background signal observed by de Boer et al. (2005), where any possible baryonic matter within the ring must be largely hidden from the observer by self-shielding effects.

Baryonic dark matter in the form of cold, dense molecular gas clumps (clumpuscules), associated with the HI disk, was proposed in Pfenniger \& Combes (1994) and Pfenniger et al. (1994). Such clumps are hardly detectable optically or with radio telescopes. They are self-shielding against $\gamma$-ray emission if their diameters are sufficiently small, e.g. $\$ 10$ AU for a clump mass of $10^{-3} M_{\odot}$ (Kalberla et al. 1999; Ohishi et al. 2004). Excess $\gamma$-ray emission, correlated with local gas and dust was found by Grenier et al. (2005). These investigations suggest that some "dark gas" is associated with H I gas, distributed like an envelope around CO-traced molecular gas. The total mass of this "dark gas" is comparable to the mass of the molecular gas, negligible in comparison to the dark matter ring.

\subsection{Origin of the dark matter ring and disk}

Our mass model is axisymmetric. However, the derived flaring shows significant asymmetries (Figs. 18 to 20). Spiral structure exists out to $R \sim 30 \mathrm{kpc}$, and we find strong radial variations and a pronounced north-south asymmetry. Typical scales for systematic fluctuations appear to be in the range 3 to $15 \mathrm{kpc}$.

The Milky Way is not a unique galaxy. Rotation curves of other galaxies show similar wiggles; perhaps the most impressive collection is given by Sofue et al. (1999). It appears hard to explain all these fluctuations as caused by an extended dark matter halo. The current understanding is that the halos represent a rather smooth distribution. Despite density enhancements at the center (cusps), no substructures on kpc scale are expected. Clumpyness appears instead to be associated with dissipative matter (Combes 2002).

The dark matter ring may provide a key to understanding these fluctuations. The ring, located at $13 \lesssim R \lesssim 18.5 \mathrm{kpc}$, has a $F W H M$ extension of $12 \mathrm{kpc}$. Associated stellar streams appear to be more sharply bounded in distance, in FWHM typically $2 \mathrm{kpc}$ to $4 \mathrm{kpc}$ (Rocha-Pinto et al. 2003; Martínez-Delgado et al. 2005). Newberg et al. (2002) determine an upper limit of $6 \mathrm{kpc}$. Simulations of the accretion of a dwarf onto the Galaxy show that the most prominent tidal arms are also spatially welldefined (Martin et al. 2005).

If the giant stellar ring is caused by the disruption of a dwarf galaxy, it appears reasonable to also associate the dark matter ring with the dwarf. The mass-over-light ratio for the progenitor galaxy would be in the range 24 to 120 , with the dark matter distribution more extended by a factor of 4 . This case resembles the Sagittarius dwarf, as modeled by Ibata et al. (1997) and Ibata \& Lewis (1998). These authors needed a similar dark matter halo to stabilize the dwarf against the Galactic tides.

Helmi \& White (1999) argue that the stellar halo may have been built up by disrupted satellites. Their calculations suggest that the stellar halo could consist of 300 to 500 streams. Assuming that these streams are associated with dark matter would imply a more or less smooth spatial distribution of dark matter debris within the Galaxy. The hypothesis that satellites may have considerable amounts of dark matter is supported 
by Gilmore et al. (2006), who argue that Milky Way dwarf spheroidals are dark matter dominated by halos extending possibly to kpc scales with typical mass-to-light ratios of a few hundreds. Hayashi et al. (2003) essentially come to the same conclusion when simulating the structural evolution of substructures. From dissipation-less simulations on structure formation, a roundish halo is expected (Allgood et al. 2006). The dark matter disk advocated by us implies a strongly anisotropic distribution for the progenitors of the stellar streams. The orbits for the majority of the accreted dwarfs must have been highly correlated with the plane of the Milky Way disk.

Willman et al. (2004) discuss the completeness of the census of Milky Way companions with respect to Galactic latitudes. For a random distribution, they estimate an incompleteness as large as $33 \%$. Up to 10 satellites at low latitudes, possibly causing 300 to 500 streams, if captured, could be waiting for detection or are already captured by the Galaxy. The recent detection of five new Milky Way companions (Belokurov et al. 2007) implies that this is only a lower limit. Abadi et al. (2003) demonstrate for disk galaxies that the bulk $(\sim 60 \%)$ of the thick disk consists of the tidal debris from satellites whose orbital plane was coincident with the disk and whose orbits were circularized by dynamical friction prior to full disruption. If these satellites contained also dark matter, one would expect that this material would have built up a thick disk-like dark matter component. The shape of the resulting dark matter disk would depend on the accretion history and on the nature of the dark matter.

Acknowledgements. P. Kalberla and L. Dedes acknowledge financial support from the Deutsche Forschungsgemeinschaft, grant KA1265/5-1, U. Haud from the Estonian Science Foundation grant no. 6106. A previous version of this paper was submitted to ApJ, but rejected. We have taken the comments of the anonymous referee then into account and thank $\mathrm{C}$. Heiles for encouraging comments in the early stages. The project got re-animated after getting knowledge of the dark matter ring hypothesis (de Boer et al. 2005), for which we thank G. Józsa and F. Kenn. We thank L. Blitz and the A\&A referee for constructive criticism, K.S. de Boer and C. Vlahakis for a critical reading of the manuscript, J. Brand, S. Malhotra, and J. Wouterloot for making observational results available.

\section{References}

Abadi, M. G., Navarro, J. F., Steinmetz, M., \& Eke, V. R. 2003, ApJ, 591, 499 Allgood, B., Flores, R. A., Primack, J. R., et al. 2006, MNRAS, 367, 1781 Bahcall, J. N., \& Soneira, R. M. 1980, ApJS, 44, 73 Bahcall, J. N. 1984, ApJ, 276, 156

Bajaja, E., Arnal, E. M., Larrarte, J. J., et al. 2005, A\&A, 440, 767

Becquaert, J.-F., \& Combes, F. 1997, A\&A, 325, 41

Bellazzini, M., Ibata, R., Martin, N., et al. 2006, MNRAS, 366, 865

Belokurov, V., Zucker, D. B., Evans, N. W., et al. 2007, ApJ, 654, 897

Benjamin, R. A. 2002, Seeing Through the Dust: The Detection of HI and the Exploration of the ISM in Galaxies, ASP Conf. Ser., 276, 201

Bergström, L., Edsjö, J., Gustafsson, M., \& Salati, P. 2006, J. Cosmol. AstroPart. Phys., 5, 6

Binney, J., \& Dehnen, W. 1997, MNRAS, 287, L5

Binney, J., \& Merrifield, M. 1998 (Princeton, NJ: Princeton University Press) Blitz, L. 1979, ApJ, 231, L115

Blitz, L., \& Spergel, D. N. 1991, ApJ, 370, 205

Brand, J., \& Blitz, L. 1993, A\&A, 275, 67

Bronfman, L., Cohen, R. S., Alvarez, H., May, J., \& Thaddeus, P. 1988, ApJ, 324,248

Burton, W. B., \& Te Lintel Hekkert, P. 1986, A\&AS, 65, 427

Burton, W. B., Elmegreen, B. G., \& Genzel, R. 1992, The galactic interstellar medium (Springer-Verlag), 412

Caldwell, J. A. R., \& Ostriker, J. P. 1981, ApJ, 251, 61

Celnik, W., Rohlfs, K., \& Braunsfurth, E. 1979, A\&A, 76, 24

Chiba, M., \& Beers, T. C. 2000, AJ, 119, 2843
Combes, F. 2002, New Astron. Rev., 46, 755

Collins, J. A., Benjamin, R. A., \& Rand, R. J. 2002, BAAS, 34, 709

de Boer, K. S., \& Savage, B. D. 1983, ApJ, 265, 210

de Boer, W., Sander, C., Zhukov, V., Gladyshev, A. V., \& Kazakov, D. I. 2005, A\&A, 444, 51

Dehnen, W., \& Binney, J. 1998, MNRAS, 294, 429

Dickey, J. M., \& Lockman, F. J. 1990, ARA\&A, 28, 215

Diplas, A., \& Savage, B. D. 1991, ApJ, 377, 126

Dubinski, J., \& Carlberg, R. G. 1991, ApJ, 378, 496

Evans, N. W., Gyuk, G., Turner, M. S., \& Binney, J. 1998, ApJ, 501, L45

Fich, M., Blitz, L., \& Stark, A. A. 1989, ApJ, 342, 272

Fichtel, C. E., Bertsch, D. L., Chiang, J., et al. 1994, ApJS, 94, 551

Fraternali, F., Oosterloo, T., Sancisi, R., \& van Moorsel, G. 2001, ApJ, 562, L47

Fraternali, F., Oosterloo, T., \& Sancisi, R. 2004, A\&A, 424, 485

Fraternali, F., Oosterloo, T. A., Sancisi, R., \& Swaters, R. 2005, Extra-Planar Gas, ASP Conf. Ser., 331, 239

Fraternali, F., \& Binney, J. J. 2006, MNRAS, 366, 449

Georgelin, Y. M., \& Georgelin, Y. P. 1976, A\&A, 49, 57

Gilmore, G., et al. 2006, Nucl. Phys. B, in press

[arXiv: astro-ph/0608528]

Grenier, I. A., Casandjian, J.-M., \& Terrier, R. 2005, Science, 307, 1292

Gyuk, G., Flynn, C., \& Evans, N. W. 1999, ApJ, 521, 190

Hartmann, D., \& Burton, W. B. 1997, Atlas of Galactic Neutral Hydrogen (Cambridge: Cambridge University Press)

Haslam, C. G. T., Salter, C. J., Stoffel, H., \& Wilson, W. E. 1982, A\&AS, 47, 1 Haud, U., \& Einasto, J. 1989, A\&A, 223, 95

Hayashi, E., Navarro, J. F., Taylor, J. E., Stadel, J., \& Quinn, T. 2003, ApJ, 584, 541

Heald, G. H., Rand, R. J., Benjamin, R. A., Collins, J. A., \& Bland-Hawthorn, J. 2006a, ApJ, 636, 181

Heald, G. H., Rand, R. J., Benjamin, R. A., \& Bershady, M. A. 2006b, ApJ, 647, 1018

Helmi, A., \& White, S. D. M. 1999, MNRAS, 307, 495

Henderson, A. P., Jackson, P. D., \& Kerr, F. J. 1982, ApJ, 263, 116

Holmberg, J., \& Flynn, C. 2000, MNRAS, 313, 209

Holmberg, J., \& Flynn, C. 2004, MNRAS, 352, 440

Honma, M., \& Sofue, Y. 1997, PASJ, 49, 453

Ibata, R. A., \& Lewis, G. F. 1998, ApJ, 500, 575

Ibata, R. A., Wyse, R. F. G., Gilmore, G., Irwin, M. J., \& Suntzeff, N. B. 1997, AJ, 113, 634

Ibata, R. A., Irwin, M. J., Lewis, G. F., Ferguson, A. M. N., \& Tanvir, N. 2003, MNRAS, 340, L21

Kalberla, P. M. W. 2003, ApJ, 588, 805

Kalberla, P. M. W., \& Kerp, J. 1998, A\&A, 339, 745

Kalberla, P. M. W., \& Haud, U. 2006, A\&A, 455, 481

Kalberla, P. M. W., Westphalen, G., Mebold, U., Hartmann, D., \& Burton, W. B. 1998, A\&A, 332, L61

Kalberla, P. M. W., Shchekinov, Y. A., \& Dettmar, R.-J. 1999, A\&A, 350, L9

Kalberla, P. M. W., Burton, W. B., Hartmann, D., et al. 2005, A\&A, 440, 775

Klypin, A., Zhao, H., \& Somerville, R. S. 2002, ApJ, 573, 597

Korchagin, V. I., Girard, T. M., Borkova, T. V., Dinescu, D. I., \& van Altena, W. F. 2003, AJ, 126, 2896

Kuijken, K., \& Gilmore, G. 1989, MNRAS, 239, 651

Kuijken, K., \& Gilmore, G. 1991, ApJ, 367, L9

Levine, E. S., Blitz, L., \& Heiles, C. 2006a, ApJ, 643, 881

Levine, E. S., Blitz, L., \& Heiles, C. 2006b, Science, 312, 1773

Lockman, F. J., \& Gehman, C. S. 1991, ApJ, 382, 182

López-Corredoira, M. 2006, MNRAS, 369, 1911

López-Corredoira, M., Cabrera-Lavers, A., Garzón, F., \& Hammersley, P. L. 2002, A\&A, 394, 883

Malhotra, S. 1994, ApJ, 433, 687

Malhotra, S. 1995, ApJ, 448, 138

Martin, N. F., Ibata, R. A., Conn, B. C., et al. 2005, MNRAS, 362, 906

Martínez-Delgado, D., Butler, D. J., Rix, H.-W., et al. 2005, ApJ, 633, 205

Merrifield, M. R. 1992, AJ, 103, 1552

Momany, Y., Zaggia, S. R., Bonifacio, P., et al. 2004, A\&A, 421, L29

Momany, Y., Zaggia, S., Gilmore, G., et al. 2006, A\&A, 451, 515

Nakanishi, H., \& Sofue, Y. 2003, PASJ, 55, 191

Narayan, C. A., Sha, K., \& Jog, C. J. 2005, A\&A, 440, 523

Navarro, J. F., Frenk, C. S., \& White, S. D. M. 1996, ApJ, 462, 563

Newberg, H. J., et al. 2002, ApJ, 569, 245

Ohishi, M., Mori, M., \& Walker, M. 2004, ApJ, 610, 868

Olling, R. P. 1995, AJ, 110, 591

Olling, R. P., \& Merrifield, M. R. 1998, MNRAS, 297, 943

Olling, R. P., \& Merrifield, M. R. 2000, MNRAS, 311, 361

Olling, R. P., \& Merrifield, M. R. 2001, MNRAS, 326, 164

Parker, E. N. 1966, ApJ, 145, 811

Parker, E. N. 1969, Space Sci. Rev., 9, 651 
Peñarrubia, J., Martinez-Delgado, D., Rix, H. W., et al. 2005, ApJ, 626, 128 Peñarrubia, J., Benson, A. J., Martínez-Delgado, D., \& Rix, H. W. 2006, ApJ, 645,240

Pfenniger, D., \& Combes, F. 1994, A\&A, 285, 94

Pfenniger, D., Combes, F., \& Martinet, L. 1994, A\&A, 285, 79

Pietz, J., Kerp, J., Kalberla, P. M. W., et al. 1998, A\&A, 332, 55

Press, W. H., Flannery, B. P., \& Teukolsky, S. A. 1986 (Cambridge: University Press)

Rand, R. J. 1997, ApJ, 474, 129

Rand, R. J. 2000, ApJ, 537, L13

Reynolds, R. J. 1997, in The Physics of Galactic Halos, ed. H. Lesch, R.-J. Dettmar, U. Mebold, \& R. Schlickeiser (Berlin: Verlag), 57

Rix, H.-W., \& Rieke, M. J. 1993, ApJ, 418, 123

Rocha-Pinto, H. J., Majewski, S. R., Skrutskie, M. F., \& Crane, J. D. 2003, ApJ, 594, L115

Rohlfs, K., \& Kreitschmann, J. 1981, Ap\&SS, 79, 289

Rohlfs, K., \& Kreitschmann, J. 1988, A\&A, 201, 51

Saha, K., \& Jog, C. J. 2006, MNRAS, 367, 1297

Savage, B. D., Sembach, K. R., \& Lu, L. 1997, AJ, 113, 2158

Schmidt, M. 1956, Bull. Astron. Inst. Netherlands, 13, 15

Schneider, S. E., \& Terzian, Y. 1983, ApJ, 274, L61

Siegel, M. H., Majewski, S. R., Reid, I. N., \& Thompson, I. B. 2002, ApJ, 578, 151
Sofue, Y., \& Rubin, V. 2001, ARA\&A, 39, 137

Sofue, Y., Tutui, Y., Honma, M., et al. 1999, ApJ, 523, 136

Spitzer, L. 1942, ApJ, 95, 329

Swaters, R. A., Sancisi, R., \& van der Hulst, J. M. 1997, ApJ, 491, 140

van Woerden, H., Wakker, B. P., Schwarz, U. J., \& de Boer, K. S. 2004, ASSL 312: High Velocity Clouds

Voskes, T. 1999, M.Sc. Thesis, University of Leiden [arXiv: astro-ph/0601653]

Weinberg, M. D. 1998, MNRAS, 299, 499

Weinberg, M. D., \& Blitz, L. 2006, ApJ, 641, L33

Weiner, B. J., \& Sellwood, J. A. 1999, ApJ, 524, 112

Westerhout, G. 1957, Bull. Astron. Inst. Netherlands, 13, 201

Willman, B., Governato, F., Dalcanton, J. J., Reed, D., \& Quinn, T. 2004, MNRAS, 353, 639

Wolfire, M. G., McKee, C. F., Hollenbach, D., \& Tielens, A. G. G. M. 2003, ApJ, 587,278

Wouterloot, J. G. A., Brand, J., Burton, W. B., \& Kwee, K. K. 1990, A\&A, 230, 21

Yanny, B., Newberg, H. J., Grebel, E. K., et al. 2003, ApJ, 588, 824

Zaritsky, D. 1999, The Third Stromlo Symposium: The Galactic Halo, ASP Conf. Ser., 165,34 